\title{
L'Allemagne, l'élargissement européen et la politique de voisinage.
}

Entre multilatéralisme et bilatéralisme

Germany, the Enlargement and Neighbourhood Policies of the European Union

Between Multilateralism and Bilateralism

\section{Elsa Tulmets}

\section{OpenEdition}

\section{Journals}

Édition électronique

URL : http://journals.openedition.org/transcontinentales/488

DOI : 10.4000/transcontinentales.488

ISBN : 978-2-8218-1408-0

ISSN : 1775-397X

\section{Éditeur}

Editions de la maison des sciences de l'homme

Édition imprimée

Date de publication : 30 juin 2006

Pagination : 121-149

ISBN : 2200-92169-1

ISSN : 1950-1684

\section{Référence électronique}

Elsa Tulmets, «L'Allemagne, l'élargissement européen et la politique de voisinage. », Transcontinentales

[En ligne], 2 | 2006, document 9, mis en ligne le 30 septembre 2012, consulté le 08 septembre 2020

URL : http://journals.openedition.org/transcontinentales/488 ; DOI : https://doi.org/10.4000/ transcontinentales. 488

Ce document a été généré automatiquement le 8 septembre 2020

Tous droits réservés 


\title{
L'Allemagne, l'élargissement européen et la politique de voisinage.
}

\author{
Entre multilatéralisme et bilatéralisme \\ Germany, the Enlargement and Neighbourhood Policies of the European Union \\ Between Multilateralism and Bilateralism
}

\section{Elsa Tulmets}

1 La chute du mur de Berlin en novembre 1989 et la réunification allemande en octobre 1990 ont suscité, en France notamment, de nombreux questionnements sur la place de l'Allemagne et de sa politique étrangère en Europe. Allait-elle, comme le craignaient certains analystes, affirmer de nouveau son rôle sur la scène internationale ${ }^{1}$ alors que, depuis la fin de la Seconde Guerre mondiale, elle avait été contenue par les structures multilatérales euro-atlantiques comme la Communauté européenne (CE), l'Union européenne (UE) et l'Organisation du traité de l'Atlantique nord (OTAN) ${ }^{2}$ ? L'engagement allemand pour un élargis-sement rapide de la Communauté européenne aux pays d'Europe centrale et orientale redevenus indépendants a été freiné par les positions plus prudentes d'autres pays membres, comme la France qui préférait privilégier le renforcement institutionnel de la CE avant -l'adhésion d'autres États. Malgré les différents points de vue défendus par les États membres de l'UE, notamment par l'Allemagne et la France, les compromis politiques successifs obtenus aux Conseils de Copenhague (1993), de Luxembourg (1997) et d'Helsinki (1999) ont permis l'adhésion en mai 2004 de huit pays d'Europe cen-trale et orientale (PECO) ${ }^{3}$ et de deux pays méditerranéens ${ }^{4}$. À partir de 1999, les implications de la future position géographique de l'Union européenne ont fait naître nombre d'interrogations : en effet, en ouvrant les premières négociations d'adhésion lors du Conseil d'Helsinki, les États membres ont accepté de repousser les frontières de l'UE aux marges de l'Ukraine, de la Biélorussie et de la Moldavie, et ils ont accordé le statut de candidat à la Turquie. Face à cette nouvelle donne géographique impliquant de confier la responsabilité des contrôles aux frontières aux nouveaux États membres et, après les événements du 11 septembre 2001, marquant 
l'interpénétration de la sécurité intérieure et extérieure, les questions sécuritaires sont devenues centrales pour plusieurs États membres. Il s'agissait de déterminer comment l'Union pouvait redéfinir les relations avec ses nouveaux voisins en sachant qu'elle n'était pas prête, politiquement, à s'élargir indéfiniment ni, institutionnellement, à assurer la sécurité dans son environnement direct. Devant les candidatures potentielles de l'Ukraine et de la Moldavie, jugées plus problématiques que celles d'Europe centrale, et jugeant nécessaire de redéfinir ses relations avec la Russie, le Conseil de l'UE a proposé en 2002 une politique de l'«Europe plus large» (Wider Europe). En 2003, afin de montrer que cette politique repré-sentait clairement une alternative à l'adhésion, la Commission a publié un concept de mise en œuvre englobant l'ensem-ble des voisins de l'Union élargie. L'initiative, officiellement adoptée par le Conseil en 2004, a été rebaptisée «Politique européenne de voisinage » (PEV) pour inclure les pays du bassin méditerranéen, certains pays du Proche-Orient ${ }^{5}$ - qui n'ont pas vocation à l'adhésion - et, plus récemment, les trois pays du Caucase du Sud (Arménie, Azerbaïdjan, Géorgie). D’une certaine façon, cette politique entend éviter de réitérer l'expérience des promesses anticipées accordées à la Turquie et proposer un cadre de coopération renforcé avec les pays aux frontières directes de l'UE ou limitrophes des pays candidats à l'adhésion.

2 Le cas de l'Allemagne, un des pays les plus engagés politiquement et financièrement dans ces politiques, est intéressant à bien des égards. Plusieurs travaux ont montré que, depuis la fin de la Seconde Guerre mondiale, la politique étrangère allemande - amputée de pouvoir militaire - avait pris la forme d'un "pouvoir civil », c'est-à-dire d'une politique s'appuyant sur des ressources comme la diplomatie, l'économie, le droit et le génie civil pour assurer la sécurité et la stabilité à l'étranger ${ }^{6}$. Cette démarche est également très proche de celle qui est défendue par les États nordiques, neutres en matière de politique extérieure. Les politiques d'élargissement et de voisinage sont toutes deux fondées sur la recherche de compromis politiques entre États membres et de solutions administratives de mise en œuvre assez similaires où, en l'absence de capacités militaires suffisantes, le pouvoir «doux» est privilégié sur le pouvoir «dur " ${ }^{7}$. Ces politiques permettent par ailleurs de comprendre de quelle manière les usages du multilatéralisme européen conduisent à la création de politiques européennes qui agissent en retour sur les préférences et les structures de politique extérieure des États membres, ce que les politologues et internationalistes spécialistes des politiques européennes appellent " européanisation " ${ }^{8}$. Élément peu souligné jusqu'ici, ce processus offre également la possibilité aux États membres d'élargir leurs champs d'action dans des domaines soutenus par les autres États de l'UE. Il tend à se multiplier dans la politique extérieure de l'Union depuis la création en 1992 de la Politique extérieure et de sécurité commune (PESC), et l'introduction de la conditionnalité ainsi que de nouveaux instruments politiques pour gérer les relations avec les États tiers.

3 En analysant comment les gouvernements successifs allemands ont participé à l'élaboration et à la mise en œuvre des politiques d'élargissement et de voisinage de l'Union européenne, cet article soulignera aussi comment le soutien allemand de l'élargissement à l'Est a permis d'accroître l'intérêt pour l'Europe centrale et orientale parmi les Quinze, de perfectionner les outils de la politique extérieure (d'élargissement, de voisinage) et, en exerçant un impact retour sur la politique allemande, d'ouvrir de nouvelles opportunités d'action pour ce pays ${ }^{9}$. Loin de représenter uniquement une contrainte, le multilatéralisme spécifique développé pour assurer la paix en Europe représenterait-il désormais une occasion pour les États membres de redéployer leur 
politique extérieure suivant une logique multi-bilatérale ? De brèves comparaisons avec le cas français mettront en valeur les ressources que le multi-latéralisme européen ouvre désormais à l'Allemagne et aux autres États membres en matière de politique extérieure. Conçu à l'origine par les Pères fondateurs comme une contrainte pour les États membres, il est désormais perçu par la Commission comme un soft power externe.

Figure 1

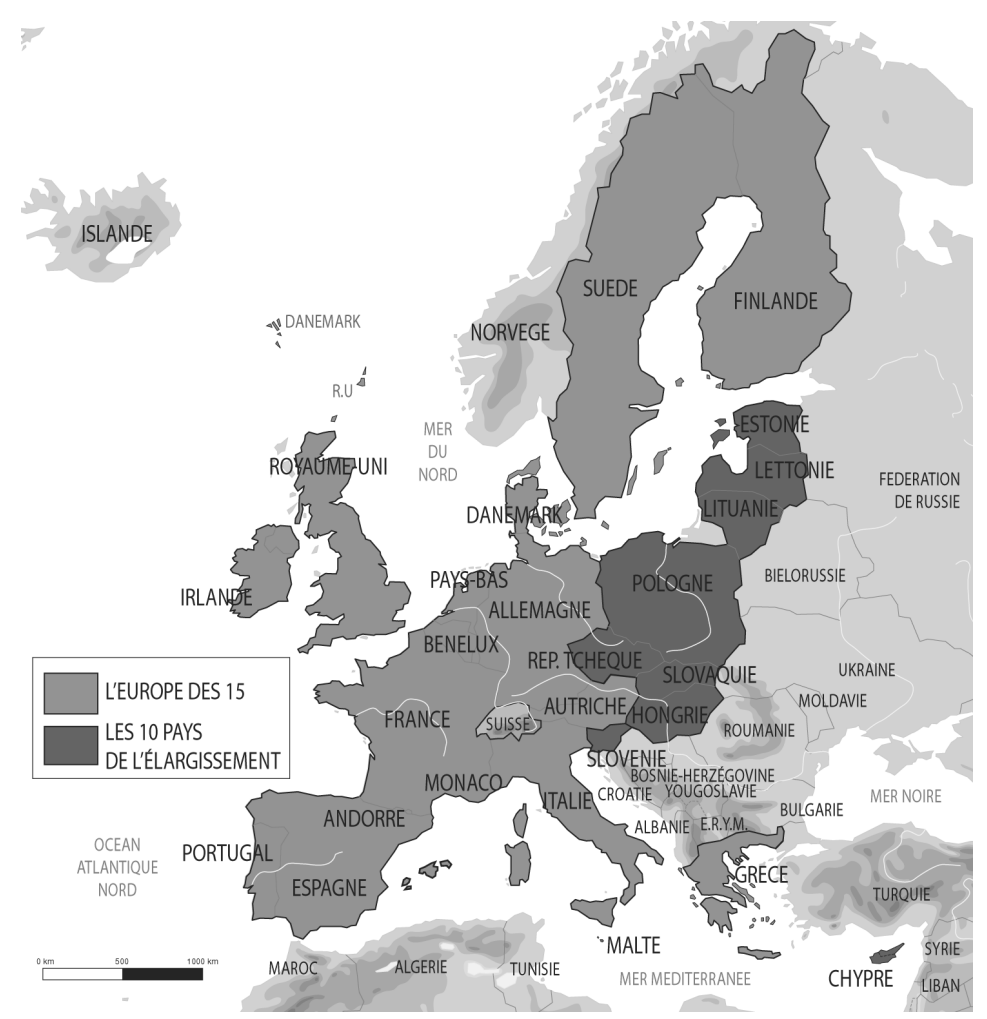

L'élargissement de l'Union européenne

\section{L'Allemagne, avocat de l'élargissement de l'Union européenne à l'Est}

En 1989-1990, la crainte de voir la Communauté européenne se diluer après le retour de l'«Occident kidnappé ${ }^{10}$ » était fortement présente chez de nombreux hommes politiques européens, et la période de 1989-1993 a souvent été qualifiée de période d'incertitude ${ }^{11}$. Dans ce contexte, les évolutions possibles de la politique étrangère de l'Allemagne réunifiée, en particulier à l'égard des PECO, n'ont cessé de susciter inquiétudes et débats parmi les autres États membres de la CE ${ }^{12}$. Entre 1989 et 1991, les hommes politiques français et allemands ont joué un rôle central pour s'accorder sur le lancement d'une politique européenne d'élargissement.

\section{Le compromis sur des préférences communes : les conditions d'adhésion}

La position engagée de l'Allemagne dans le renforcement des relations avec l'Europe centrale et orientale puise ses fondements dans la politique allemande d'après-guerre 
menée par le chancelier Konrad Adenauer (Union démocrate chrétienne, CDU), mais surtout dans celle qu'initia à partir des années 1970 l'ancien maire de Berlin devenu chancelier, Willy Brandt (Parti social démocrate d'Allemagne, SPD). Cette politique, restée célèbre sous le nom de "nouvelle Ostpolitik», avait principalement pour but de voir un jour disparaître la division entre les deux Allemagnes. Le gouvernement allemand démocrate-chrétien des années 1980 (CDU-CSU) - soutenu par les groupes d'intérêts économiques, mais aussi par les associations d'alle-mands expulsés d'Europe centrale - s'est également engagé dans les négociations entre la Communauté européenne et le Conseil d'assistance économique mutuelle (CAEM), qui ont abouti à la signature de la «Déclaration conjointe portant sur la coopération et la reconnaissance mutuelle » du 25 juin 1988. Cette déclaration est particulièrement importante dans le sens où elle met un terme à la période de non-reconnaissance mutuelle entre les deux blocs économiques.

\section{Legs du passé et partage du fardeau}

6 Peu après les événements de la fin de l'année 1989, le gouvernement de la République fédérale d'Allemagne (RFA) a commencé à soutenir l'idée d'un élargissement de la Communauté européenne aux anciens pays socialistes redevenus souverains. Les travaux sur les discours allemands ont montré que la rhétorique de l'élite politicoadministrative vis-à-vis des PECO s'est construite jusqu'à la fin des années 1990 autour de quatre idées principales: la gratitude, la responsabilité, le multilatéralisme et la solidarité ${ }^{13}$. La gratitude doit être comprise comme étant liée à la réunification allemande. En effet, l'Ostpolitik allemande développée depuis les années 1970 tendait tout entière au règlement de la question nationale, c'est-à-dire à la réunification des deux Allemagnes et, par là-même, des deux «Europes " ${ }^{14}$. Comme l'indiquait Klaus Kinkel, ministre des Affaires étrangères de la RFA, devant le Parlement allemand peu après la chute du mur de Berlin : «sans la volonté de liberté de nos voisins et amis de l'Est, nous n'aurions pas pu atteindre notre but national le plus important, la réunification ${ }^{15}$ ». Les discours politiques à partir de 1989 reflètent sans exception la dimension historique des relations avec l'Europe centrale et orientale et la responsabilité que l'Allemagne nazie a joué dans la division de l'Europe et le destin de ces pays. Ils indiquent la volonté du gouvernement de la République fédérale d'Allemagne d'aider financièrement et de manière différenciée l'ensemble des pays postcommunistes - et plus spécifiquement les pays d'Europe centrale comme la Pologne, la Hongrie et, à l'époque encore, la Tchécoslovaquie - afin de les soutenir dans leur volonté de se rapprocher des structures euro-atlantiques. Les premiers discours insistent pour que cette aide se réalise dans un cadre multilatéral, celui d'organisations comme la Conférence/Organisation pour la sécurité et la coopération en Europe (CSCE/ OSCE), le Conseil de l'Europe, l'Organisation du traité de l'Atlantique nord (OTAN) et surtout la Communauté européenne (CE). Ces objectifs figuraient déjà en bonne place dans les programmes électoraux de la CDU et du FDP (Parti libéral) à la veille des élections de décembre $1990^{16}$. En appelant les autres pays de la Communauté à rejoindre l'Allemagne dans l'effort d'assistance accordée à l'Europe centrale, ils indiquaient clairement que la politique extérieure allemande entendait continuer, après 1989, à se définir dans un cadre multilatéral et européen.

D'autres motivations ayant trait à des raisons de politique intérieure - en matière économique, environnementale, migratoire et sécuritaire - expliquent le 
positionnement rapide du gouvernement démocrate-chrétien en faveur d'un élargissement de la CE et de la recherche d'une solution fondée sur la solidarité et le multi-latéralisme. Les principaux groupes d'intérêt économiques allemands, fortement intéressés par les marchés d'Europe centrale et orientale en raison de la longue présence d'entreprises allemandes à l'Est, ont constamment soutenu cette politique. Parmi ces groupes d'intérêt, on retrouve en premier lieu la Fédération de l'industrie allemande (BDI), en particulier son Comité Est (Ost-Ausschuß) qui joua un rôle spécifique de para-diplomatie lors de l'Ostpolitik initiée par le chancelier Willy Brandt ${ }^{17}$. En 1995, le gouvernement était particulièrement pressé d'annoncer que les entreprises allemandes exportaient plus vers l'ancien Bloc soviétique que vers les États-Unis ${ }^{18}$. La proximité des frontières polonaise et tchèque a facilité les coopérations pour réduire les pollutions transfrontalières, en particulier dans le cadre des Eurorégions créées au cours des années 1990. Cependant, les relations bilatérales entre l'Allemagne et ses voisins polonais et tchèques restent toujours envenimées par l'activisme des associations d'expulsés allemands et la mémoire du passé, comme en attestent les débats sur la restitution des biens immobiliers, les décrets Beneš ou la création d'un "Centre contre l'expulsion» (Zentrum gegen Vertreibungen). Au niveau sécuritaire, l'arrivée massive de "rémigrés ${ }^{19}$ " venus de l'Est sur le sol allemand a obligé le gouvernement non seulement à mettre en place des mesures d'intégration de ces personnes par une politique engagée du logement et de l'enseignement de la langue allemande, mais aussi à augmenter les mesures de sécurité intérieure ${ }^{20}$. La politique de coopération économique à l'Est, tant bilatérale que multi-latérale, ainsi que l'éventualité d'une politique d'élargissement de la Communauté européenne à l'Est, représentaient pour le gouvernement allemand les seules mesures capables de diminuer cet afflux de populations sur son territoire et de réduire les coûts de leur prise en charge, qui venaient s'ajouter à ceux de la réunification allemande. On espérait qu'en soutenant l'intégration économique et politique des PECO aux Communautés européennes, ces populations resteraient dans leur pays de résidence. En obtenant le soutien d'autres États membres, le fardeau économique et migratoire porté par l'Allemagne pourrait se répartir sur le territoire de la CE/UE. Le discours sur l'engagement multilatéral de la politique allemande à l'Est a été tenu dans plusieurs enceintes institutionnelles jusqu'au lancement d'une politique européenne d'élargissement en 1993. Cette même année, Klaus Kinkel écrivait encore :

«Aucune alternative, autre que la consolidation de structures démocratiques chez nos partenaires d'Europe de l'Est, ne pourra mieux renforcer la sécurité et la stabilité de l'Europe. Il y va de l'intérêt vital de l'Allemagne qui, à cet égard, se retrouve à nouveau au premier rang et ne peut relever seule ces nouveaux défis. Elle supporte, il est vrai, une charge supérieure à la moyenne dans la stabilisation de l'Europe de l'Est, mais elle a atteint les limites de ses capacités. Elle a maintenant besoin de l'appui de tous ses alliés. Ce que l'Allemagne peut accomplir en Europe de l'Est, elle ne sera pas en mesure d'y parvenir avec le soutien de ses partenaires occidentaux, ni sans eux, ni surtout contre eux..$^{21}$ ”

\section{Multilatéralisme et pouvoir civil}

8 L'engagement allemand envers les pays d'Europe centrale et orientale - traduit dans les discours politiques par le rôle d'«avocat" des PECO - fut, à partir de 1989, constamment réitéré. Le rétablissement de la stabilité politique et économique à l'Est et le maintien de la sécurité en Europe n'ont cessé de représenter les priorités de la 
politique allemande, tant au sein du SPD ${ }^{22}$ que de la CDU. En 1994, le programme politique de la CDU, rejoignant le rapport Schäuble-Lamers, précisait que l'Allemagne :

«a un intérêt particulier au développement durable, démocratique et pacifique des pays d'Europe centrale, orientale et du Sud-Est. En raison de la position centrale de l'Allemagne, il est de notre intérêt politique, économique et sécuritaire que nos voisins de l'Est trouvent une stabilité comparable à celle de l'Europe de l'Ouest. Nous voulons contribuer à assurer leur construction démocratique, économique, sociale et écologique. Sinon de lourdes conséquences sont à prévoir en retour pour l'Allemagne et pour toute l'Europe. La coopération avec ces États est donc un point essentiel de notre politique étrangère ${ }^{23}$."

9 Ainsi, pour Roman Herzog, ancien président allemand, les changements survenus en Europe depuis 1989 ont obligé le gouvernement allemand à redéfinir sa politique extérieure dans une vision plus globale, tout en maintenant son ancrage à l'Ouest, en usant de son influence économique et morale plutôt que militaire, et en se fixant pour devoir d'unir l'Europe ${ }^{24}$. Dans l'esprit des hommes politiques allemands de l'époque, la stabilité aux frontières et la sécurité ne pouvaient être assurées que par des moyens civils, et la réalisation de réformes à l'Est devait être encouragée par le pouvoir d'attraction de l'Union européenne et en particulier par l'adhésion.

Dans son discours du 31 décembre 1989, le président François Mitterrand effectuait une contre-proposition à l'élargissement : son projet, aux motivations multiples ${ }^{25}$, consistait à renforcer les relations avec l'Europe centrale et orientale par la création d'une "Confédération européenne ${ }^{26}$ " englobant les PECO, tout en travaillant à renforcer l'intégration interne de l'Europe des Douze. Le président français justifiait son programme par la nécessité de trouver une solution européenne à un contexte européen sans l'aide des États-Unis, de contrôler la résurgence de sentiments identitaires minoritaires susceptibles de déstabiliser l'Europe et d'encadrer le rôle d'une Allemagne en voie de réunification dans une Europe ayant vocation à s'élargir. Car il était nécessaire, dans l'esprit de l'élite politique au pouvoir, d'offrir une proposition française qui permette de contrebalancer le rôle de l'Allemagne réunie en Europe. Les discussions sur l'intégration économique et monétaire ainsi que sur l'Europe militaire et politique - qui ont abouti au Traité de Maastricht (1992) - ont notamment eu pour but -d'engager et de lier encore plus l'Allemagne à la construction de l'Union européenne ${ }^{27}$. Par ailleurs, économiquement, la France commençait à s'inquiéter de l'importance des investissements allemands à l'Est et à réaliser que les PECO représentaient des marchés moins risqués que prévu ${ }^{28}$. Par le biais de la MICECO, puis des Cocop ${ }^{29}$, le gouvernement français choisit de mettre l'accent sur sa politique économique et culturelle, en particulier en Pologne, Hongrie et Roumanie.

11 Ainsi peut-on dire que, politiquement, les débats sur l'élargissement et l'approfondissement du projet européen, et plus largement sur la définition d'une identité européenne, se sont cristallisés autour du désaccord franco-allemand sur la finalité de l'Union ${ }^{30}$. Dès 1989, l'argument allemand en faveur de l'élargissement de la Communauté européenne à l'Est trouve écho auprès d'autres États membres, en particulier de l'Italie et des Pays-Bas. La Grande-Bretagne ainsi que le Danemark expriment également peu de réticences à l'encontre d'une adhésion rapide de certains PECO à la Communauté européenne, une position qui s'explique par la volonté de ne pas voir les institutions européennes renforcées, mais au contraire diluées dans un espace de libre-échange toujours plus large. Présentant l'avantage d'arrimer ces nouveaux membres plus rapidement à la démocratie, comme cela avait été le cas pour l'Espagne, le Portugal et la Grèce, ces propositions sont toutefois jugées irréalistes par 
les économistes, qui mettent en chœur l'accent sur le faible degré de développement de ces pays et leur inexpérience en matière d'économie de marché. Au début des années 1990, un second groupe d'États prend ainsi position contre un élargissement rapide de la Communauté à l'Est. Des pays comme l'Espagne, la Grèce et le Portugal se méfient de l'arrivée de nouveaux membres qui constitueraient des concurrents sérieux pour l'obtention des fonds structurels communautaires. La Belgique considère tout nouvel élargissement précipité comme un obstacle à l'approfondissement des institutions communautaires. En tête de file se trouve la France, qui craint que l'extension à l'Est ne confère à l'Allemagne un poids politique et économique prépondérant en Europe, et qui redoute aussi une dilution des institutions et politiques européennes, comme la politique agricole commune (PAC), dans un espace élargi de libre-échange.

12 Face aux diverses positions, les unes jugées trop romantiques, les autres trop libérales, le président François Mitterrand et le gouvernement français ont joué un rôle central de contre-balancier à la politique allemande et de frein à la mise en place d'une politique rapide d'élargissement de la Communauté européenne ${ }^{31}$. En 1993, le débat se concentre sur la recherche d'une solution aux défis posés par la demande de candidature de certains PECO. Ici encore, le multilatéralisme représente une ressource à laquelle recourt cette fois le gouvernement français :

«La responsabilité de la Communauté et de ses membres est donc de préparer cette adhésion en mettant en place les mécanismes qui la rendront possible [...]. [La France] demande, par ma voix, devant vous, ce matin, à la Communauté d'établir, avant 1993, un rapport relatant pays par pays, les moyens à réunir pour rendre l'adhésion possible et les délais nécessaires pour y parvenir; tout cela, bien entendu, dans le respect des principes communautaires qui s'imposent à tous. ${ }^{32}$ "

13 Un compromis est finalement scellé lors du Sommet de Copenhague de 1993. Le Conseil valide la proposition de la Commission, élaborée à partir de l'expérience des élargissements précédents et des positions des États membres, d'accepter -l'adhésion de nouveaux candidats européens à condition que ceux-ci aient rempli un certain nombre de critères ${ }^{33}$. Ces conditions - qui font référence à la mobilisation de valeurs et de ressources civiles - représentent désormais la base de l'identité européenne et du pouvoir d'attraction européen ${ }^{34}$ :

\begin{tabular}{|l|l|}
\hline $\begin{array}{l}\text { Critère } \\
\text { politique }\end{array}$ & $\begin{array}{l}\text { L'adhésion requiert de la part du pays candidat qu'il ait des institutions stables } \\
\text { garantissant la démocratie, la primauté du droit, les droits de l'homme, le respect } \\
\text { des minorités et leur protection. }\end{array}$ \\
\hline $\begin{array}{l}\text { Critère } \\
\text { économique }\end{array}$ & $\begin{array}{l}\text { L'adhésion nécessite l'existence d'une économie de marché viable ainsi que la } \\
\text { capacité de faire face à la pression concurrentielle et aux forces du marché à } \\
\text { l'intérieur de l'Union. }\end{array}$ \\
\hline $\begin{array}{l}\text { Critère } \\
\text { juridique } \\
\text { de reprise de } \\
\text { l'acquis }\end{array}$ & $\begin{array}{l}\text { L'adhésion présuppose la capacité du pays candidat à en } \\
\text { assumer les obligations, et notamment à souscrire aux objectifs de l'union } \\
\text { politique, économique et monétaire. }\end{array}$ \\
\hline
\end{tabular}

14 En 1994, la présidence allemande du Conseil s'est fixé pour objectif principal de concrétiser la déclaration politique de 1993 et a joué un rôle central pour définir une 
stratégie de pré-adhésion qui permette le rapprochement politique, économique et juridique des candidats à l'Union européenne. Cette stratégie, annoncée lors du Conseil d'Essen, regroupe différents instruments de mise en œuvre :

(certains bénéfices économiques ou politiques sont suspendus ou supprimés si les États receveurs ne respectent pas certaines normes et valeurs) est complétée par la conditionnalité positive fondée sur la solidarité (plus les réformes relatives à la reprise de l'acquis sont mises en œuvre, plus les États candidats reçoivent une aide encourageant à mettre en œuvre ces réformes) ${ }^{37}$. Cependant, entre 1996 et 1997, la Commission réalise que ces formes classiques de conditionnalité ne fonctionnent pas comme elle l'aurait voulu. Elle décide d'introduire une réforme qui tienne compte a) du contexte politique national et de l'engagement politique des gouvernements pour la réalisation des réformes; b) du renforcement des capacités institutionnelles, administratives et judiciaires des pays receveurs pour accroître la mise en œuvre des réformes $^{38}$. Le recours aux instruments de la nouvelle gestion publique et à l'expertise des États membres en matière de mise en œuvre de l'acquis (jumelages institutionnels introduits en 1997) devait ainsi permettre de développer la capacité de l'UE à contrôler la réelle mise en œuvre de l'acquis communautaire dans les pays candidats, mais aussi de reconstruire la légitimité interne de la Commission (critiques de la Cour des comptes, du Parlement européen, crise de 1999) ainsi que la légitimité externe de la politique d'élargissement, jugée unilatérale et asymétrique.

\section{Une « européanisation » de la politique allemande ?}

La «nouvelle méthode » que la Commission propose en 1997 dans son « Agenda 2000 », complétée par la suite dans divers documents de stratégie ${ }^{39}$, s'inspire étrangement de la «méthode ouverte de coordination » dont les caractéristiques avaient déjà été définies en 1997 lors du Sommet européen pour l'emploi (voir tableau comparatif page 141). En proposant une alternative à la conditionnalité classique, cette méthode, appliquée à l'élargissement, devait permettre la définition d'engagements communs matérialisés par des buts à atteindre (ou benchmarks) inscrits dans un contrat, le «Partenariat pour l'adhésion». Dans l'esprit de la Commission, la rhétorique du partenariat l'autorisait, en tant que gardienne des traités et donc de la conditionnalité, à faire aussi peser la responsabilité politique et financière du respect des conditions d'adhésion sur les candidats et à assurer ainsi la légitimité de cette politique. Dans ce sens, il est possible de parler de "conditionnalité négociée " entre les parties au contrat ${ }^{40}$. Tandis que la méthode communautaire adaptée à l'élargissement n'offrait pas de possibilité de sanction (la Cour de justice des CE ne pouvait pas être mobilisée tant que les candidats n'étaient pas membres), la "nouvelle » méthode ouvrait des moyens de contrôle du respect des critères d'adhésion en rappelant aux candidats leurs 
engagements grâce aux évaluations par les pairs (experts des États membres) et aux rapports annuels de la Commission (naming and shaming: dénonciation et humiliation par la Commission en cas de non-mise en œuvre).

\section{Du programme allemand Transform au jumelage institutionnel PHARE}

Conformément à cette méthode, les États membres ont eu besoin de mieux se coordonner pour proposer des «modèles " européens à reprendre et pour adapter leurs politiques nationales aux règles définies par la Commission. La décision d'engager une politique européenne d'élargissement à l'Est a en effet été suivie d'un phénomène d'« européanisation » des politiques nationales qui s'est traduit par un changement des discours politiques (vers 1994), puis par une nouvelle organisation de la coopération extérieure des États membres (vers 1998). L'exemple du programme bilatéral allemand Transform, créé en 1992 pour soutenir la démocratisation et la transition vers l'économie de marché en Europe centrale et orientale, est assez parlant. Conçu comme un moyen de «compenser la faible participation des acteurs allemands aux programmes PHARE et $\mathrm{TACIS}^{41}$ » alors que le gouvernement contribuait à près $\mathrm{d}^{\prime}$ '« un tiers de leur budget total ${ }^{42}$ ", le discours sur le transfert de l'économie sociale de marché, du fédéralisme et de l'expérience de la réunification allemande a progressivement fait place à partir de 1994-1995 à celui sur l'aide à la reprise de l'acquis communautaire ${ }^{43}$ et des normes de l'Union européenne. À la même période, le budget (national) du programme Transform commençait à diminuer et les organisations de mise en œuvre postulaient déjà pour des projets européens leur permettant de poursuivre leurs activités. La création des jumelages institutionnels PHARE ${ }^{44}$ en 1997 ainsi que des programmes ISPA (fonds structurels) ${ }^{45}$ et SAPARD (politique agricole) ${ }^{46}$ en 1998 ont notamment forcé les États membres à s'adapter aux procédures imposées par la Commission en introduisant les méthodes de la nouvelle gestion publique dans leur fonctionnement interne. Par ailleurs, un «Point de contact national » a été créé au ministère des Finances - chargé de coordonner les politiques européennes depuis le changement gouvernemental de 1998 - pour faciliter la communication avec Bruxelles. Ainsi, non seulement le discours politique a évolué, mais aussi les structures de mise en œuvre de la politique allemande à l'Est. L'interprétation des préférences allemandes au niveau européen s'est donc répercuté sur les discours politiques, les institutions et les organisations de la politique étrangère allemande. Une fois que ces préférences nationales sont réadoptées au niveau européen, les États membres qui les ont promues n'en gardent donc plus toute la maitrise. C'est également ce qu'indique le développement parallèle de la politique méditerranéenne.

\section{Participation parallèle au Partenariat euro-méditerranéen}

Phénomène souvent oublié aujourd'hui, le lancement officiel en 1993 de la politique d'élargissement à l'Est a donné lieu à l'ouverture d'un débat largement repris par la littérature académique ${ }^{47}$ sur les relations de l'Union européenne avec les autres pays tiers, non candidats à l'adhésion. Les accords économiques signés avec les PECO n'ont cessé d'être comparés à ceux conclus avec les pays du bassin méditerranéen et avec les pays Afrique-Caraïbe-Pacifique (ACP) (généralement d'anciennes colonies de la GrandeBretagne, de la France et de la Belgique). Afin de compenser l'engagement de l'Union à l'Est et de recentrer les priorités de politique extérieure de l'UE vers le Sud, la France, l'Espagne et l'Italie ont fait de la coopération avec les pays méditerranéens une des 
questions centrales de la présidence espagnole de 1995. Le Sommet de Madrid a ainsi inauguré une nouvelle coopération régionale, le Partenariat euro-méditerranéen (ou Processus de Barcelone), pour relancer les relations avec les pays d'Afrique du Nord et compenser l'engagement de l'UE vers l'Est ${ }^{48}$. En 2000, alors que le Partenariat s'essoufflait, l'Union européenne a introduit une méthode similaire à celle de l'élargissement pour mieux coordonner les politiques des États membres. Certains acteurs allemands non gouvernementaux comme la Fondation Bertelsmann, think tank du gouvernement financé par l'entreprise du même nom, ont joué un rôle important de conseil auprès de la Commission pour soutenir cette méthode ${ }^{49}$. La création d'une véritable politique européenne portée par des pays comme la France, puis la mobilisation d'instruments analogues à la méthode ouverte de coordination, ont eu pour résultat d'exercer graduellement un impact d'« européanisation » des structures de coopération des États membres, même les plus engagés comme la France ${ }^{50}$.

\section{Construction de la problématique de sécurité aux frontières de l'UE élargie}

23 La réalisation de l'espace Schengen et la communautarisation des politiques de visas en 1997 ont également contribué à repousser les frontières intérieures de l'Union et à y introduire une nouvelle conception de la sécurité. En effet, pour l'Allemagne par exemple, la sécurité intérieure ne se définit plus aux frontières avec ses voisins directs, mais au niveau des gares et aéroports français, espagnols et italiens pour le Sud, et bientôt lituaniens, polonais, hongrois ou slovènes pour l'Est et le Sud-Est. Au fil des Sommets européens de la fin des années 1990, on voit donc se développer parmi les États membres une manière décloisonnée de concevoir leurs intérêts sécuritaires pour y intégrer des aires régionales où ils ne sont pas traditionnellement présents. Les manières de percevoir les risques ont fini par se rejoindre, en particulier à la fin de l'année 2001 : ces risques, relevant de la sécurité «soft » et non-militaire (immigration, trafics, voire terrorisme) entrent dans le domaine de la sécurité nationale dès qu'ils pénètrent le territoire de l'Union, ne serait-ce que par un seul État membre. Ainsi, la prise en compte d'autres aires régionales dans la politique extérieure des États membres, comme l'Europe de l'Est en France ou la Méditerranée en Allemagne, résulte essentiellement des avancées du processus d'intégration européenne ${ }^{51}$.

24 En 1998, au moment où débutent les négociations d'adhésion, des interrogations semblables à celles de l'après 1989 réapparaissent dans l'agenda européen: quelle politique adopter vis-à-vis des futurs voisins de l'Union élargie? L'UE a-t-elle les capacités d'affronter un nouvel élargissement et les moyens d'une politique innovante à l'égard de ses voisins ? Cette problématique est l'une des quatre thématiques traitées par les études conjointes du centre d'analyse et de prévision du ministère français des Affaires étrangères et du Plannungsstab de l'Auswärtiges Amt, transmises lors des Sommets franco-allemands entre novembre 1998 et juin 2000 (études intitulées « L'Europe à trente États membres et plus ») ${ }^{52}$. La question des frontières de l'Europe a fait l'objet d'un rapport en octobre 1999 qui mentionnait déjà les difficultés que l'Union devrait affronter dans un avenir proche. Ce rapport mettait en lumière toute la difficulté que les liens, alors encore relativement ténus entre ces deux pays et la Russie, pourraient poser à l'UE. Les experts conseillaient ainsi au couple franco-allemand de ne pas réveiller les susceptibilités russes par un éventuel isolement de Moscou et de faire en sorte que l'Ukraine et la Biélorussie ne retombent pas dans la sphère de dépendance russe. En Allemagne, la Fondation Bertelsmann - écoutée par la Commission - a également 
organisé en 2001 des rencontres d'experts pour discuter du «futur de l'Europe ». Elle formula plusieurs recommandations et insista sur la nécessité d'inclure les candidats aux réflexions sur les politiques européennes ${ }^{53}$. Les discussions à ce sujet se sont poursuivies dans des cercles politiques et universitaires plus ou moins fermés. En décembre 2002, un peu moins de dix ans après le lancement de la politique d'élargissement à l'Est et au moment où cet élargissement était politiquement acquis, la présidence danoise de l'Union européenne ouvrait officiellement le débat sur l'avenir des relations avec le voisinage de l'UE.

\section{De l'élargissement vers l'Est à la Politique européenne de voisinage}

Lors des négociations d'adhésion lancées à partir de mars 1998 avec les pays d'Europe centrale et orientale, le gouvernement social-démocrate allemand élu en septembre 1998 s'est montré plus ferme que le gouvernement précédent sur certains dossiers sensibles représentant des thématiques électorales centrales, comme l'immigration et la sécurité. L'Allemagne et l'Autriche ont obtenu des périodes de transition étendues s'agissant de la question de la libre circulation des travailleurs et de certains domaines Schengen. La thématique des risques aux frontières de l'Union élargie, due à l'arrivée de nouveaux voisins et au doublement de la frontière avec la Russie, a également été soutenue et développée par les pays nordiques et la Grande-Bretagne. Engagé dans ce débat, le gouvernement social-démocrate allemand a défendu une approche plus favorable au renforcement des relations de l'UE avec la Russie qu'avec les voisins directs de l'Union européenne élargie. L'inclusion dans la politique de la Wider Europe rebaptisée "Politique de voisinage» en 2003 - d'autres pays que ceux de la Communauté des États indépendants (CEI) comme les pays du bassin méditerranéen et ceux du Caucase du Sud semble désormais multiplier les opportunités d'action extérieure pour les États membres. Nous nous demanderons dans quelle mesure la politique de voisinage, résolument européenne dans le sens où elle reflète l'agrégation des préférences hétérogènes de ses États membres, a ouvert à l'Allemagne et à d'autres États membres la possibilité d'élargir le champ de leur politique étrangère dans des régions où ils ne sont pas traditionnellement présents.

\section{Origines de la Wider Europe et nouvelle méthode dans l'action extérieure européenne}

Les travaux sur l'origine de la politique de voisinage étant quasiment inexistants, un ensemble d'hypothèses peuvent être avancées sur la naissance de cette politique qui reste étroitement liée au contexte du cinquième élargissement de l'Union européenne ${ }^{54}$. Plusieurs groupes d'acteurs ont contribué en premier lieu à la mise sur agenda de la stratégie de la Wider Europe. La Commission a commencé dès le milieu des années 1990 à faire pression sur les pays candidats pour signer des traités de «bon voisinage » avec leurs voisins directs. Ces références ont été de plus en plus fréquentes dans les rapports annuels de la Commission avec la communautarisation de la politique de Justice et Affaires intérieures en 1997 (en particulier politique des visas), indiquant que la gestion des frontières extérieures de l'UE élargie devait être renforcée. Après l'adhésion à l'Union, en 1995, de l'Autriche, de la Suède et de la Finlande, le contrôle aux frontières a 
pris une importance particulière, notamment en raison de la longue frontière commune entre la Finlande et la Russie $(1300 \mathrm{~km})$. Il n'est donc pas étonnant de constater qu'en 1997, le Premier ministre finlandais Paavo Lipponen proposait d'enrichir la politique extérieure de l'UE par une «dimension nordique» pour renforcer la coopération dans des domaines clés comme «les infrastructures, l'environnement et les ressources naturelles, la sécurité nucléaire, la santé publique, le commerce, la lutte contre le crime organisé, la corruption et le terrorisme " 55. Pour les pays nordiques, cette initiative avait notamment pour but de créer une aire de coopération sous-régionale au Nord de l'Europe qui viendrait contrebalancer le Processus de Barcelone. Chacune de ces politiques était déjà guidée par l'idée d'éviter, grâce à une méthode souple de coopération, la création de nouvelles lignes de partage entre États membres et pays voisins, qu'il s'agisse des pays du Nord de l'Europe concernés par les relations avec l'Est ou de ceux du Sud inquiétés par les différences croissantes avec les pays de la Méditerranée et par les conflits latents dans cette région. La stabilisation de la situation politique et économique dans les Balkans ainsi que le dépôt de la candidature croate à l'adhésion en 2003 puis de l'ancienne république yougoslave de Macédoine en 2004 ont incité l'Union européenne à proposer une réponse cohérente pour l'ensemble de ses futurs nouveaux voisins afin d'instaurer et de gérer dans chacune de ces aires géographiques «la stabilité et la sécurité » aux frontières extérieures de l'UE.

\section{Une position allemande en faveur du renforcement des liens avec la Russie}

La situation géographique de l'Allemagne et les discours politiques sur sa responsabilité historique envers l'Europe centrale et orientale ont permis au gouvernement allemand de développer une politique particulièrement engagée d'assistance aux anciens pays de l'URSS, en particulier à la Russie, l'Ukraine et la Biélorussie, pays prioritaires dans le programme Transform (près d'un tiers du budget) ${ }^{56}$. L'accord germano-russe du 16 juillet 1990 avait accordé à l'URSS un crédit de cinq milliards de Deutsche Mark et l'accord sur les conditions financières du retrait des troupes soviétiques prévoyait le versement de douze milliards de Deutsche Mark, plus un crédit sans intérêts de trois milliards de Deutsche Mark ${ }^{57}$. L'engagement bilatéral allemand s'avérant insuffisant, le chancelier Helmut Kohl a mené une politique active visant à mobiliser les ressources de plusieurs enceintes multilatérales. En 1989, il appelait l'ensemble des pays de l'OCDE à fournir une assistance commune et coordonnée, et à renforcer l'association de l'URSS au processus de coopération en Europe ${ }^{58}$. Le chancelier allemand obtint des Douze une aide alimentaire lors du Conseil européen de Rome en décembre 1990, et il plaida en juillet 1991 lors du G7 pour que l'URSS soit admise à part entière au FMI et à la Banque mondiale. Au niveau sub-régional, le gouvernement allemand a participé activement au renforcement de la coopération baltique-nordique ${ }^{59}$ et à celle des pays du Danube ${ }^{60}$. En raison de l'importance de la Russie dans la politique extérieure allemande, le gouvernement social-démocrate mené par le chancelier Gerhard Schröder a encouragé en 2003 la création d'une politique européenne à l'égard des voisins orientaux de l'Union élargie, cette fois principalement pour des raisons liées aux politiques d'énergie et de sécurité ${ }^{61}$. En 2001, des experts européens rassemblés par la Fondation Bertelsmann proposaient de lancer une politique de la "dimension est-européenne " entre États membres, candidats et tiers en s'inspirant de la "dimension nordique ", des projets de coopération transfrontière et de l'expérience de l'élargissement, tout en 
incluant les instruments déjà existants comme l'Accord de partenariat et de coopération ainsi que le programme TACIS ${ }^{62}$.

\section{Une stratégie européenne pour assurer la sécurité à l'Est, puis dans l'ensemble du} voisinage

La politique à l'égard des nouveaux voisins contenait une dimension nettement plus sécuritaire que l'élargissement et nécessitait un rattachement à la Politique extérieure et de sécurité commune (PESC). En avril 2002, le Conseil des affaires générales demande au haut représentant pour la PESC, Javier Solana, et à la Commission, représentée par Christopher Patten, commissaire pour les relations extérieures, de prendre ensemble position à ce sujet. La lettre commune Solana-Patten publiée en août 2002 marque la nécessité de mobiliser les trois piliers de l'Union européenne pour mettre en œuvre une politique qui touche donc aussi bien les politiques du premier pilier (économie, agriculture, environnement...) que celles des deux autres piliers, la PESC et la coopération en matière de justice et affaires intérieures (JAI). La lettre indique cependant des hésitations quant aux ensembles géographiques à inclure. En décembre 2002, Romano Prodi pose les jalons de la politique de la Wider Europe de l'Union européenne en offrant de partager "plus qu'un partenariat et moins que les institutions » de l'UE ${ }^{63}$. Il s'agit clairement dans ce contexte d'éviter un nouveau "cas turc » où l'Union s'engagerait dans des promesses qu'elle ne pourrait peut-être pas tenir, en particulier vis-à-vis de l'Ukraine et de la Moldavie. Les pays des Balkans, mentionnés dans la lettre commune sont finalement exclus de l'initiative en 2003 en raison de la spécificité de leur contexte politique et du dépôt de la candidature croate à l'adhésion : l'UE décide de leur consacrer une politique sous-régionale spécifique ${ }^{64}$.

En mars 2003, la Commission communique sa proposition au Conseil pour donner corps à la politique de la Wider Europe, entre temps renommée politique de "nouveau voisinage " pour y inclure d'autres pays que ceux situés à l'Est ${ }^{65}$. Plusieurs pays candidats à l'adhésion, comme la Pologne, les États baltes (en particulier la Lituanie et l'Estonie) ainsi que la Roumanie ont réagi positivement à l'initiative de Bruxelles. Le gouvernement russe de Vladimir Poutine refuse, quant à lui, d'être inclus dans la stratégie européenne et négocie en mai 2003, au Sommet de Saint-Pétersbourg, un partenariat spécial avec l'Union européenne comprenant quatre domaines de coopération prioritaires ou "espaces communs». Le contour de ces quatre espaces a été défini lors du Sommet de La Hague de novembre 2004 dans des "feuilles de route" spécifiques ${ }^{66}$ que le gouvernement allemand avait notamment proposées ${ }^{67}$.

Sous la pression des pays du bassin méditerranéen membres de l'UE, en particulier de la France et de l'Espagne, le débat sur la redéfinition des relations avec les voisins de l'Est s'est élargi aux relations avec ceux du Processus de Barcelone. Lancé en 1995, ce processus commençait à s'essouffler pour les mêmes raisons que le progra-mme PHARE: le partenariat économique était jugé unilatéral, au profit des États membres, et la conditionnalité classique montrait déjà ses limites ${ }^{68}$. L'élargissement de l'initiative de la Wider Europe aux pays méditerranéens et du Proche-Orient offrait ainsi l'opportunité à l'UE de redéfinir son rôle dans cette aire géographique. En mars 2003, la Commission a donc inclus les pays de la coopération méditerranéenne et du programme MEDA ${ }^{69}$ à sa proposition, acceptée en 2004 par le Conseil. Le 12 mai 2004, la Commission proposait un «Papier de stratégie » et des « Rapports par pays » afin de mieux définir les priorités que l'Union européenne et ses États membres s'engageaient à poursuivre. En décembre 2004, 
la Commission présentait le nouveau document de coopération différenciée appelé «Plan d'action " ${ }^{70}$. La politique prenait alors définitivement le nom de "Politique européenne de voisinage " (PEV) et devenait une des composantes principales de la «Stratégie européenne de sécurité $»$ de J. Solana ${ }^{71}$.

\section{Les instruments du soft power}

31 Afin d'organiser la coordination et la cohérence de cette nouvelle politique, la Commission s'est principalement appuyée sur l'expérience de la réforme de la politique d'élargissement à l'Est (« Agenda $2000 », 1997)$ en définissant une rhétorique et des instruments assez semblables. Plusieurs fonctionnaires de la DG Élargissement ayant participé à la rédaction des différentes propositions au Conseil ont avoué sans détour avoir copié quasiment à l'identique les documents développés à partir de 1997 pour la politique d'élargissement et les avoir adaptés progressivement au contexte du nouveau voisinage ${ }^{72}$. Cette approche a été complétée par un volet sécuritaire permettant l'inclusion de la gestion civile des crises, un domaine où la Commission essaie progressivement d'élargir ses compétences ${ }^{73}$.

Ainsi, on retrouve au niveau rhétorique presque tous les éléments des conditions d'adhésion à l'Union européenne. Sous le manteau du «respect des valeurs communes » se cachent en effet :

«[...] le respect des droits de l'homme, incluant le droit des minorités, l'État de droit, la bonne gouvernance, la promotion des relations de bon voisinage, et les principes de l'économie de marché et du développement durable ainsi que certaines priorités de politique étrangère ${ }^{74} »$.

La conditionnalité classique définie dans les accords de partenariat et d'association des pays TACIS et MEDA a été complétée en 2000-2001 par l'approche négociée développée pour l'élargissement à l'Est. Entre mai et décembre 2004, la Commission s'est appuyée sur l'expérience des «Partenariats pour l'adhésion» afin de proposer les premiers «Plans d'action par pays » avec les voisins ayant manifesté un intérêt pour la politique de voisinage ${ }^{75}$. Le respect des engagements communs et de la réalisation des réformes sont assurés par des mécanismes d'évaluation (rapport annuels) et de contrôle par la Commission et par les pairs. Cette méthode a donc pour but d'accroître la cohérence interne de la politique européenne ${ }^{76}$, et d'augmenter sa légitimité, son acceptation et son appropriation par les pays voisins afin d'assurer « la stabilité, la croissance, le bienêtre et la sécurité » tout autour de l'Union européenne.

La politique de voisinage, en réalisant le lien entre les trois piliers de l'UE communautaire, de sécurité intérieure et extérieure (soft et hard security) - représente donc un moyen pour l'Union de tester sa capacité à devenir un acteur inter-national cohérent non seulement par l'exportation de ses normes, mais aussi par la gestion des crises présentes dans son entourage immédiat. Contrairement à l'élargissement, où la question sécuritaire a été encadrée par le transfert du régime Schengen à l'Est et par l'adhésion des PECO à l'OTAN, la redéfinition de relations de coopération et de stabilité avec les nouveaux voisins était plus préoccupante. En effet, plusieurs pays - dont la majorité n'a pas vocation à l'adhésion - présentent des situations de crises internes ou régionales dont la proximité géographique est perçue comme une menace à la stabilité de l'Union. La Commission mentionne notamment les situations tendues en Transnistrie (Moldavie), au Sahara occidental (Maroc), au Proche-Orient, mais aussi, plus récemment, dans le Caucase du Sud ${ }^{77}$. Après la «Révolution des roses » qui a 
apporté un changement politique important en Géorgie, le Conseil a également accepté en avril 2005 la proposition de la Commission d'intégrer l'Arménie, l'Azerbaïdjan et la Géorgie à la Politique de voisinage.

Tableau 1

\begin{tabular}{|c|c|c|}
\hline $\begin{array}{l}\text { MOC } \\
\text { (Politique de I'emploi, } \\
\text { Luxembourg 1997) }\end{array}$ & $\begin{array}{c}\text { Politique } \\
\text { d'élargissement } \\
\text { («Agenda2000», 1997) }\end{array}$ & $\begin{array}{l}\text { Politique de voisinage } \\
\text { (Communication de } \\
\text { la Commission, 2003) }\end{array}$ \\
\hline (a) Objectifs européens & Critères d'adhésion & $\begin{array}{l}\text { Engagements sur des valeurs } \\
\text { communes }\end{array}$ \\
\hline (b) Plans d'action nationoux & $\begin{array}{l}\text { Partenariat pour l'adhésion } \\
\text { Plan national d'adoption } \\
\text { de lacquis (PNAA) (plan élaboré } \\
\text { par les candidats précisant } \\
\text { les réformes à suive } \\
\text { pour reprendre l'acquis) } \\
\text { Plan d'action pour le renforcement } \\
\text { des capacités administrotives } \\
\text { et judiciaires }\end{array}$ & $\begin{array}{l}\text { Plans d'action } \\
\text { (élaborés en commun) } \\
\text { Papiers de stratégie par pays } \\
\text { Plans de suivi } \\
\text { de la mise en ceruvre }\end{array}$ \\
\hline $\begin{array}{l}\text { (c) Cycles de politique annuels } \\
\text { ou bi-annuels }\end{array}$ & $\begin{array}{l}\text { Cycles de négociation, } \\
\text { programmation de l'assistance }\end{array}$ & $\begin{array}{l}\text { Plans d'action ( } 3 \text { à } 5 \text { ans), } \\
\text { programmation multi-annuelle } \\
\text { de l'assistance (TACIS, MEDA } \\
\text { et. après } 2007 \text {, instrument } \\
\text { de la Politique de voisinage } \\
\text { et de partenariat) }\end{array}$ \\
\hline $\begin{array}{l}\text { (d) Buts à atteindre/échanges de } \\
\text { bonnes pratiques }\end{array}$ & $\begin{array}{l}\text { Buts ò atteindre (benchmarks) } \\
\text { dans le PNAA et les contrats } \\
\text { de jumelage/échanges } \\
\text { de bonnes pratiques } \\
\text { par les jumelages }\end{array}$ & $\begin{array}{l}\text { Buts à afteindre (benchmarks) } \\
\text { dans les plans d'action } \\
\text { et les contrats de jumelage }\end{array}$ \\
\hline $\begin{array}{l}\text { (e) Participotion, consultation } \\
\text { des partenaires sociaux }\end{array}$ & $\begin{array}{l}\text { Consultation des partenaires } \\
\text { sociaux au niveau national } \\
\text { et de I'UE (Commission) }\end{array}$ & $\begin{array}{l}\text { Participation au niveou national } \\
\text { et des programmes } \\
\text { communautaires, coopération } \\
\text { de epersonnes à personnes: }\end{array}$ \\
\hline $\begin{array}{l}\text { (f) Indicateurs lorsque l'acquis } \\
\text { ne le précise pas }\end{array}$ & $\begin{array}{l}\text { Listes des DG de la Commission } \\
\text { (acartes routières } x \text { ), contrats } \\
\text { de jumelage }\end{array}$ & $\begin{array}{l}\text { Listes d'acquis foumies } \\
\text { par les DG de la Commission. } \\
\text { contrats de jumelages }\end{array}$ \\
\hline $\begin{array}{l}\text { (g) Rapports de progrès } \\
\text { par la Commission }\end{array}$ & $\begin{array}{l}\text { Rapports annuels } \\
\text { par la Commission }\end{array}$ & $\begin{array}{l}\text { Rapports par pays } \\
\text { de la Commission }\end{array}$ \\
\hline
\end{tabular}

Sources : Tableau réalisé à partir de : Conclusions du Conseil européen sur l'emploi du 20-21 novembre 1997 ; Commission européenne, « Agenda 2000, Pour une Union plus forte et plus large », COM (97) 2000 final, Bulletin de l'UE, Supplément 5/97, 1997 ; Commission européenne, « Vers I'Union élargie. Document de stratégie et Rapport sur les progrès réalisés par chacun des pays candidats sur la voie de l'adhésion », COM (2002) 700 final, 2002 ; Commission européenne, « Wider Europe - Neighbourhood. A New Framework for Relations with our Eastern and Southern Neighbours », COM (2003) 104 final, 2003.

\section{La Politique européenne de voisinage, un moyen d'élargir l'action extérieure de l'Allemagne?}

Derrière l'apparente cohérence de cette politique, qui se veut et s'affiche particulièrement innovante, se cachent cependant encore de nombreux dysfonctionnements, en terme de coordination, auxquels ni les institutions européennes ni les États membres de l'UE n'ont encore trouvé de solution. Résultant de la réunion des intérêts géographiques de l'ensemble des membres de l'Union européenne, la politique de voisinage reste encore structurée par aires régionales et s'appuie souvent sur des partenariats déjà existants. En privilégiant une logique de différenciation visant à mieux considérer les paramètres nationaux des pays voisins, cette politique tente désormais d'adopter une manière contextualisée d'établir un " cercle d'amis » socialisés aux valeurs, normes et standards européens. Cette approche différenciée laisse encore libre cours à l'expression de préférences nationales très variées. L'assouplissement de la méthode a cependant ouvert de nouvelles possibilités 
de coopération entre États membres dans des domaines ou des régions qui ne font pas traditionnellement partie de leurs priorités de politique extérieure.

\section{La formation de coalitions entre l'Allemagne et les nouveaux États membres}

Cette approche, développée à l'origine pour solliciter la convergence de vues entre pays membres et candidats, allie de plus en plus anciens et nouveaux membres. Certaines coopérations ont commencé avant même l'élargissement officiel au $1^{\text {er }}$ mai $2004^{78}$. En 2001, plusieurs experts invités par la Fondation Bertelsmann recommandaient déjà à la Commission d'encourager la participation des États candidats d'Europe centrale et orientale aux réflexions sur la politique extérieure de l'Union européenne, en particulier sur les relations avec les nouveaux voisins ${ }^{79}$. Au niveau bilatéral, les initiatives germano-polonaises vis-à-vis de l'Ukraine représentent un exemple assez parlant. La Pologne a tenté à plusieurs reprises de sensibiliser les hommes politiques et les fonctionnaires européens à l'importance de ses relations avec ce pays, notamment lors des négociations sur le chapitre Justice et Affaires intérieures (introduction de visas et contrôles à la frontière orientale de la Pologne) et par la publication de papiers de position sur la politique à l'Est. L'expérience de la coopération germano-polonaise réalisée dans divers cadres (Transform, eurorégions, jumelages administratifs, etc.) ${ }^{80} \mathrm{a}$ notamment servi de référence pour la mise en place de structures de coopération entre Polonais et Ukrainiens. Une des initiatives les plus remarquées - un peu sur le modèle de l'étude franco-allemande «l'Europe à trente et plus » - fut la proposition commune de deux think tanks, l'un allemand (Stiftung Wissenschaft und Politik), l'autre polonais (Institut Spraw Publicznych), de former une position germano-polonaise susceptible de pousser l'Union européenne à soutenir activement le climat de réformes en Ukraine après la «Révolution orange » de l'hiver 2004-2005 ${ }^{81}$. Comme le précise le document germano-polonais, le parti social-démocrate allemand (SPD) a toujours privilégié le renforcement des relations avec la Russie, contrairement au parti démocrate chrétien (CDU) qui a également défendu la construction d'une coopération solide avec l'Ukraine, dans le cadre du programme Transform notamment. De manière générale, les intérêts allemand et polonais semblent s'être rejoints pour des raisons différentes : la Pologne conçoit l'Ukraine comme un partenaire essentiel en Europe, alors que l'Allemagne l'envisage comme un pont entre l'UE et la Russie ${ }^{82}$. Au bout du compte, la coopération germano-polonaise n'en est pas vraiment sortie renforcée : la décision prise en 2005 de construire un pipeline germano-russe en mer Baltique en contournant la Pologne a envoyé aux oubliettes les tentatives de coopération en Ukraine au niveau de l'énergie ${ }^{83}$. Pour certains analystes, la politique allemande a cependant acquis pour fonction d'ouvrir la voie à l'UE dans des domaines où la coopération multilatérale à l'Est - et en particulier avec la Russie - resterait difficile ${ }^{84}$. 
Figure 2

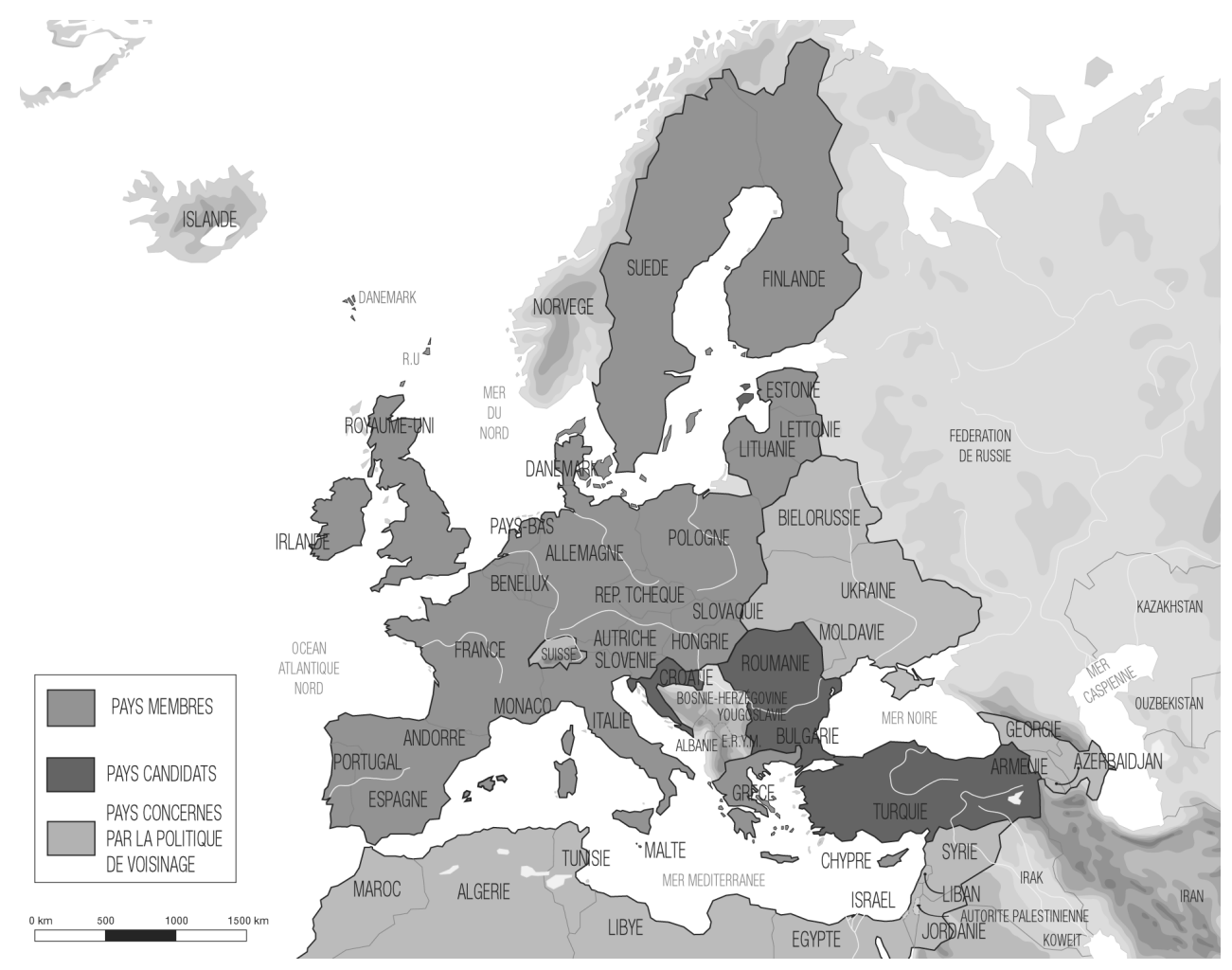

\section{Une mise en œuvre encore timide de la Politique européenne de voisinage}

37 L'initiative germano-polonaise - malgré ses faiblesses - est venue gonfler le nombre des critiques formulées à l'encontre de la non-décision de l'Union européenne de réagir aux situations d'instabilité à ses frontières. L'année 2005 a en effet été marquée par un nombre d'initiatives cruciales dans la Politique de voisinage. Le Conseil a accepté en février le Plan d'action en dix points pour l'Ukraine proposé par la commissaire Benita Ferrero-Waldner et le haut représentant pour la PESC Javier Solana. Le 23 mars 2005, l'Union a ouvert un poste de représentant spécial de l'UE en République de Moldavie sur le modèle des hauts représentants de l'OSCE. Un projet de coopération à la frontière entre l'Ukraine et la Moldavie a été lancé pour créer cinq postes-frontières afin de lutter contre des activités illégales (trafics d'armes, de drogues et de personnes) qui pourraient avoir des répercussions aux frontières de l'Union. En avril 2005, le Conseil a accepté d'intégrer les pays du Caucase du Sud à la Politique de voisinage, décision soutenue notamment par les États baltes qui ont défini une politique du « $3+3$ » axée sur la solidarité entre anciennes petites républiques soviétiques ${ }^{85}$. La mission " état de droit » EUJUST Thémis en Géorgie a été conclue avec succès en juillet 2005. Il est intéressant de constater qu'en intégrant les pays du Caucase du Sud et ceux du ProcheOrient à la Politique de voisinage, l'Union européenne essaie de former un cercle de pays « amis » s'étendant autour d'un État candidat à l'adhésion comme la Turquie. Il est possible de se demander quelles synergies pourraient émerger d'un rapprochement des politiques d'élargissement et de voisinage dans le sens où ces politiques emploient désormais des instruments de coopération relativement identiques pour faire face à des défis aux contours similaires. L'expérimentation d'une mise en œuvre par étapes, qui masque le manque de croisement jusqu'ici des expertises techniques et régionales au 
sein de la Commission ${ }^{86}$, est reflétée par l'évolution progressive du discours et des outils mobilisés pour donner corps à la Politique européenne de voisinage.

\section{Le multilatéralisme comme moyen d'élargir les sphères d'action des États membres}

Malgré l'accord sur les risques communs et les instruments mobilisés, les intérêts qui motivent les États membres restent différents en fonction des aires régionales auxquelles la politique s'adresse et des priorités définies dans les Plans d'action par pays. La politique allemande à l'Est répond par exemple à d'autres motivations que celle menée en Méditerranée. La politique française est, quant à elle, toujours nettement plus présente au Sud qu'à l'Est. Il semblerait cependant que la Politique de voisinage ait permis à l'Allemagne de légitimer et d'affirmer un peu plus son rôle en Méditerranée et que l'élargissement ait eu pour conséquence d'accroître l'intérêt de certains États membres pour l'Est, qu'il s'agisse de négociations politiques ou de la mise en œuvre de projets de coopération. Le gouvernement allemand est par exemple de plus en plus présent auprès d'États membres comme la France et la Grande-Bretagne dans la politique de l'UE au Proche-Orient. Le discours de soutien à Israël rappelle régulièrement la responsabilité allemande dans le massacre du peuple juif au cours de la Seconde Guerre mondiale ${ }^{87}$. Parallèlement, le gouvernement allemand participe à une large part de l'assistance européenne et inter-nationale aux territoires palestiniens ${ }^{88}$. Au niveau politique, l'Allemagne s'est activement engagée dans la résolution du conflit israëlo-palestinien, en particulier après l'arrivée au pouvoir de la coalition rouge-verte en 1998.

L'ancien ministre des Affaires étrangères Joschka Fischer s'est très souvent rendu dans la région et a servi de médiateur entre les parties à deux reprises en 2001, la première fois après un attentat suicide à Tel-Aviv en juin 2001, la seconde en août $2001{ }^{89}$. La publication de son « papier de stratégie » (idea paper) en 2002 avait également pour but de pousser l'Union européenne à s'impliquer un peu plus dans la résolution multilatérale du conflit ${ }^{90}$. Le gouvernement allemand a ainsi joué un rôle central dans la proposition de " feuille de route " pour la paix entre Arabes et Israéliens à côté de la France et de la Grande-Bretagne dans les négociations du «Middle-East Quartet » (États-Unis, Union européenne, Russie, Nations unies) ${ }^{91}$. Pour Volker Perthes, spécialiste de la politique allemande au Proche-Orient, « la feuille de route et le Quartet sont dans un sens les bébés du ministère des Affaires étrangères allemand, mais cela fait partie de cette politique de laisser les Américains les considérer comme leur propre création, du moment que cela aide la mise en œuvre ${ }^{92}$ ».

L'Allemagne entretient également depuis longtemps des relations privilégiées avec l'Iran et participe activement aux côtés de la France et de la Grande-Bretagne aux négociations sur la question nucléaire dans ce pays. Dans ce contexte, les analystes s'accordent pour dire que les intérêts allemands au Proche-Orient sont donc moins liés à des préoccupations économiques ${ }^{93}$ que sécuritaires et qu'ils marquent la volonté de l'Allemagne de jouer un rôle croissant dans la politique étrangère et de sécurité de l'Union européenne.

41 En termes de mise en œuvre, il est intéressant de constater que depuis l'arrêt de la Cour constitutionnelle de Karlsruhe de 1993 autorisant les troupes allemandes à participer aux opérations de maintien de la paix, les soldats allemands sont intégrés à plusieurs missions sous mandats onusien et de la Politique européenne de sécurité et de défense (PESD) dans 
les Balkans, en Méditerranée et dans le Caucase ${ }^{94}$. Cette expérience pourrait servir de référence en cas de besoin dans le cadre de la Politique de voisinage pour d'éventuelles actions PESD et projets de gestion civile des crises. Dans l'immédiat, en l'absence de réelles capacités militaires, l'UE mise sur la prévention en mobilisant des moyens civils et les politiques d'assistance pour assurer la stabilité et la sécurité dans la Politique de voisinage ${ }^{95}$. Les projets TAIEX ${ }^{96}$ et de jumelages institutionnels européens (programmes d'envoi d'experts de court et moyen terme à l'étranger) ${ }^{97}$ ouvrent ainsi la possibilité à plusieurs États membres, anciens et nouveaux, de relancer des partenariats traditionnels et parfois de s'engager dans des aires régionales où ils n'avaient, jusqu'ici, aucune tradition de coopération et donc peu de légitimité. Cette pratique avait été amorcée dans le cadre de l'élargissement à l'Est, où des pays comme l'Espagne et l'Irlande s'étaient de plus en plus affirmés dans des domaines tels que la politique régionale ou la politique de la pêche alors que les traditions de coopération avec l'Est étaient quasi inexistantes. Dans le cadre de la Politique européenne de voisinage, la Pologne observe de plus en plus près les possibilités de coopération en Méditerranée ${ }^{98}$ et la France entrevoit des moyens de travailler avec les nouveaux États membres dans les Balkans et le Caucase ${ }^{99}$. Pour l'Allemagne, l'engagement dans le Sud de l'Europe est certes encore timide, mais de plus en plus effectif. Cette présence est assurée par des moyens para-diplomatiques comme les fondations politiques ${ }^{100}$ ou des organisations chevronnées en matière de développement comme la Gesellschaft für technische Zusammenarbeit (GTZ). Le gain d'appels d'offre et de projets de jumelages européens, si minime soit-il, est chaque fois vécu par les experts comme une mini-victoire et comme un moyen de prendre pied dans une aire régionale où l'Allemagne avait jusqu'ici peu l'occasion d'offrir ses services. Des projets de jumelages ont par exemple été remportés récemment par des organisations allemandes en Tunisie ou au Maroc grâce à un montage administratif impliquant des experts français. Cette évolution, qui s'est traduite par une réelle coopération entre experts nationaux de l'UE dans le cadre de l'élargissement, risque de se faire plus rare dans le contexte du voisinage. Il s'agit en effet d'une politique où les relations historiques sont toujours très présentes et d'un domaine - l'assistance technique - relativement concurrentiel, où les enjeux économiques restent importants. Les coopérations actuellement envisagées vont plutôt dans le sens d'alliances entre nouveaux et anciens États membres, les uns disposant de l'expérience de la transition, les autres des ressources et de l'expertise régionale. Le chemin emprunté par l'Union européenne dans la politique d'élargissement à l'Est, puis dans la Politique de voisinage reflète donc la mise en place d'un réel multi-bilatéralisme européen où la politique européenne, jusqu'ici concurrencée par celle des États membres, est désormais mise en œuvre par les experts de ces États. Le multilatéralisme européen, qui avait à l'origine pour fonction de contraindre la volonté de puissance d'États souverains en les poussant à renoncer à certaines prérogatives nationales, semble aujourd'hui avoir développé d'autres fenêtres d'opportunité aussi bien au niveau de la formation de coalitions politiques que de projets de mise en œuvre. Il représente désormais, certes encore timidement, une occasion pour les États membres d'ouvrir leur politique extérieure à d'autres domaines d'activité et à d'autres aires régionales et d'enrichir ainsi les moyens pour l'Union européenne de développer sa présence internationale. 


\section{Conclusion}

Cet article a montré que la politique extérieure allemande, bien qu'ayant suscité de nombreuses craintes après l'effondrement du mur de Berlin, continue à se définir dans un contexte multilatéral et européen. La dynamique européenne, en constante redéfinition, a également gagné la politique extérieure des autres États membres de l'UE, comme en témoignent les exemples des politiques d'élargissement et de voisinage européen. La perception de risques communs à l'ensemble des États membres a entraîné la définition d'outils de coopération innovants (réforme de la politique d'élargissement en 1997, adaptation à la Politique de voisinage en 2003) qui ont, a priori, permis de développer une certaine culture du compromis dans le domaine de la politique extérieure. Cette nouvelle approche, qui tente de faire le lien entre multilatéralisme et bilatéralisme, présente la particularité de développer les outils du pilier communautaire au service de la politique étrangère de l'UE et ainsi de renforcer la cohérence interne de l'Union. L'analyse de la définition des préférences allemandes au sein de la politique d'élargissement à l'Est et de la Politique de voisinage a mis en valeur le fait que les politiques européennes contribuent à modifier les intérêts des États qui ont œuvré pour leur définition. Alors qu'au début des années 1990 la France était peu intéressée par l'Est et l'Allemagne peu engagée en Méditerranée, on constate au cours des années 1990 et 2000 une nette progression croisée au point que les investissements français à l'Est ont parfois dépassé ceux de l'Allemagne (par exemple en Pologne) et que l'Allemagne s'implique politiquement et financièrement dans la politique méditer-ranéenne et dans la résolution du conflit israélo-palestinien.

43 Les politiques d'élargissement et de voisinage sont donc complémentaires dans le sens où elles poursuivent des buts d'intégration internes et externes à l'Union européenne et mobilisent principalement des ressources de nature "civile» pour assurer la sécurité et la stabilité dans l'environnement proche de l'UE. Le multi-latéralisme développé pour assurer la paix en Europe prend aujourd'hui dans le domaine des relations extérieures une forme assez similaire au pouvoir civil pensé par l'Allemagne en l'absence de réelles capacités militaires, mais aussi par d'autres pays comme la France dans le domaine culturel. Pour preuve, les discours sur l'élargissement et le voisinage promeuvent de manière croissante le soft power de l'UE comme manière efficace de faire de la politique étrangère par les capacités d'attrac-tion et la mobilisation de moyens civils ${ }^{101}$. Loin de représenter uniquement une contrainte, le multilatéralisme européen semble désormais ouvrir la possibilité aux États membres de redéployer leur action extérieure, et de le faire ensemble dans les domaines relevant des compétences de la Commission. La forme souple de la nouvelle méthode adoptée au cours des années 1990 propose donc une réponse à l'absence de subsidiarité clairement définie pour la politique extérieure. Elle prend la forme d'un multi-bilatéralisme spécifique, notamment en matière de mise en œuvre où les ressources et l'expertise des États membres se mettent progressivement au service de la politique de l'Union européenne. Cependant, même si la nouvelle méthode de coopération extérieure contraint les États membres à "européaniser » leurs politiques, il n'est pas certain qu'elle contribue à réduire les phénomènes de concurrence entre anciens États membres au sein de la politique européenne. Par ailleurs, les discours fondés sur le pouvoir d'attraction de l'UE et ce nouveau mode de gouvernance extérieure font déjà face à plusieurs questions éthiques. En effet, les États tiers entrevoient clairement, sous 
le couvert des discours sur le partenariat et d'instruments politiques a priori plus cohérents, les traits d'un "nouvel unilatéralisme déguisé ». À l'Union européenne échoit donc la responsabilité de prouver l'efficacité réelle de cette nouvelle approche qui ne passe pas par la force, mais par la norme et la persuasion ${ }^{102}$ ainsi que de démontrer, sur le terrain, la réalité des partenariats engagés.

\section{NOTES}

1. Voir : P. Davis et P. Dombrowski, «Appetite of the Wolf: German Assistance for Central and Eastern Europe », German Politics, 6 (1), 1997, pp. 1-22 ; A. Hyde-Price, Germany and European Order : Enlarging NATO and the EU, Manchester/New York, Manchester University Press, 2000 ; C. Hacke, Die Auflenpolitik der Bundesrepublik Deutschland: Weltmacht wider Willen?, Francfort-sur-Main/ Berlin, Ullstein, 1997.

2. La thèse du "pouvoir entravé » a notamment été développée par Peter Katzenstein : Tamed Power. Germany in Europe, Ithaca, Cornell University Press, 1997

3. Dans un sens géographique, les pays de l'Europe centrale et orientale (PECO) incluent un large ensemble de pays comme les pays d'Europe centrale (Pologne, République tchèque, Slovaquie, Hongrie), les pays baltes (Estonie, Lettonie, Lituanie), les pays des Balkans (Roumanie, Bulgarie, exYougoslavie) et l'Albanie. Dans le sens défini par la Commission européenne, l'abréviation «PECO » inclut les huit candidats à l'adhésion de 2004 (Estonie, Hongrie, Lettonie, Lituanie, Pologne, République tchèque, Slovaquie, Slovénie) et les deux pays candidats à l'adhésion de 2007 (Roumanie, Bulgarie).

4. Malte et Chypre.

5. Maroc, Algérie, Tunisie, Libye, Égypte, Israël, Autorité palestinienne, Liban, Jordanie, Syrie.

6. H. Maull, «Germany and Japan : The New Civilian Powers ", Foreign Affairs, hiver 1991-1992, pp. 91-106; « Allemagne et Japon : deux pays à suivre », Politique étrangère, 2, 1995, pp. 477-496.

7. La notion de soft power a été définie par Joseph Nye pour qualifier le pouvoir d'attraction des États-Unis dans des domaines comme l'économie et la culture, par opposition au pouvoir militaire ou hard power (Joseph S. Nye, «Soft Power», Foreign Policy, 80, Fall, 1990, pp. 153-171). Cette notion est désormais très présente dans les discours des membres de la Commission européenne, notamment pour qualifier le pouvoir d'attraction de l'UE (politique, économie, droit, culture) dans son voisinage.

8. Lorsqu'il n'existe pas encore de politique définie au niveau européen, l'intégration européenne offre l'occasion aux États membres de porter leurs intérêts au niveau communautaire afin d'en faire ceux de l'Union. Ce processus, qualifié de bottom-up par la littérature anglo-saxonne en science politique, équivaut à la création de politiques européennes qui emportent ensuite, de manière hiérarchique (top-down), des adaptations au niveau national (européanisation). Sur l'européanisation : C. Lequesne et Y. Surel (dir.), L'Intégration européenne. Entre émergence institutionnelle et recomposition de l'État, Paris, Presses de Sciences Po, 2004 ; C. Radaelli, "Whither Europeanization ? Concept Stretching and Substantive Change », European Integration online Papers (EIoP), 4 (8), 2000, http://eiop.or.at/ eiop/texte/2000-008a.htm ; J. Caporaso, M. Green-Cowles et T. Risse (éds), Transforming Europe : Europeanization and Domestic Change, Ithaca, Cornell University Press, 2001. 
9. Cet article s'appuie sur les résultats de notre recherche de DEA sur la politique allemande à l'Est et surtout sur notre doctorat (E. Tulmets, «La conditionnalité dans la politique d'élargissement de l'Union européenne à l'Est: un cadre d'apprentissages et de socialisation mutuelle?», IEP Paris/FU Berlin, 2005). Les informations sur la politique de voisinage proviennent de notre recherche post-doctorale réalisée à l'Institut universitaire européen de Florence sur le transfert de l'expérience de l'élargissement vers la politique de voisinage.

10. M. Kundera, «Un occident kidnappé ou la tragédie de l'Europe centrale », Le Débat, 27, novembre 1983.

11. J. Wedel, Collision and Collusion :The Strange Case of Western Aid to Eastern Europe, 1989-1998, New York, St Martin' Press, 1998.

12. G. Lepesant, Géopolitique des frontières orientales de l'Allemagne : les implications de l'élargissement de l'Union européenne, Paris, l'Harmattan, coll. «Pays de l'Est», 1998; A.-M. Le Gloannec, «L'Allemagne, l'Europe centrale et l'Europe orientale », L'Autre Europe, 34/35, mars 1997, pp. 51-53 ; J. Sgard, «Économie: encore un miracle allemand à l'Est?», L'Autre Europe, 34/35, mars 1997, pp.64-78; J.P.Bled, «Les enjeux en Europe centrale: une perspective historique", Revue d'Allemagne, 27 (3), juillet-septembre 1995, pp.311-441 ; A. Pradetto et P. Sigmund, « Deutschland und Osteuropa in der Ära des Postkommunismus", Deutschland Archiv, 26 (8), août 1993, pp. 890-904; L.Carroué, «L'Allemagne réunifiée dans l'économie de l'Europe centrale: une hégémonie renouvelée ", Hérodote, 68, $1^{\mathrm{er}}$ trimestre 1993, pp. 83-111; H. Stark, «L'Est de l'Europe et l'Allemagne: des rapports complexes ", Politique étrangère, 56 (4), hiver 1991, pp. 859-871; A. Grosser, «L'Allemagne élargie dans l'Europe élargie », Politique étrangère, 56 (4), hiver 1991, pp. 825-832.

13. M. Ecker-Ehrhardt, « Alles nur Rhetorik? Der ideelle Vorder- und Hintergrund der deutschen Debatte über die EU-Osterweiterung", Zeitschrift für internationale Beziehungen, 9 (2), 2002, pp. 209-252.

14. A.-M. Le Gloannec, La Nation orpheline : les Allemagnes en Europe, Paris, Hachette, 1990.

15. Discours de Klaus Kinkel devant le Bundestag, 13/224, cité par M. Ecker-Ehrhardt, «The Rhetoric of Risk and Responsibility - Understanding the German Public Debate on EU Enlargement », in I. Richter, R. Müller-Schmidt et S. Berking (éds.), Risk Society and Culture of Precaution, Palgrave Macmillan (à paraître).

16. R. Fritsch-Bournazel, L'Allemagne unie dans la nouvelle Europe, Bruxelles, Complexe, 1991, pp. 322-324.

17. O. W. von Amerongen, Der Weg nach Osten, Vierzig Jahre Brückenbau für die deutsche Wirtschaft, Munich, Knaur, 1992.

18. "Deutschland exportiert mehr in den ehemaligen Ostblock als in die USA », Süddeutsche Zeitung, 19 juillet 1996 ; «La puissance commerciale allemande se renforce en Europe de l'Est. La France fait piètre figure dans la région. La domination allemande à l'Est », Le Monde, 18 juillet 1996.

19. On traduit par « rémigrés » le terme allemand Aussiedler qui désigne les personnes d'origine allemande revenues des pays d'Europe de l'Est.

20. En 1987, plus de 86000 rémigrés en provenance des anciens territoires allemands de l'Est (Pologne, 48423 personnes, et Tchécoslovaquie, 835) et d'autres régions d'Europe centrale et orientale (Roumanie, 13994 personnes, Hongrie, 581 et Union soviétique, 14 488) avaient pénétré le territoire de la RFA. Il s'agissait du chiffre le plus élevé depuis 1958, un chiffre multiplié par deux par rapport à l'année 1986; Presse- und Informationsamt der Bundesregierung, «Aussiedler-Bilanz des Jahres 1987 », Bulletin, (5), 13 janvier 1988, p. 35. En 1988, ces chiffres avaient de nouveau presque doublé, cette fois en l'espace d'un an: le gouvernement comptabilisait 140226 réfugiés en provenance de la Pologne, 47552 de l'Union soviétique, 12902 de la Roumanie et près de trois fois 1000 personnes venues de Tchécoslovaquie, de Hongrie et de 
Yougoslavie ; H. Müller, Schlaglichter der deutschen Geschichte, Bonn, Bundeszentrale für politische Bildung, 1996, p. 436.

21. K. Kinkel, "L'Allemagne en Europe", in Fondation Robert Schuman (éd.), Les nouvelles frontières de l'Europe, Paris, Economica, 1993, p. XV.22 - Après son élection en 1998, le chancelier Gerhard Schröder (SPD) affirma dans un discours d'investiture devant le Bundestag, et lors du Forum de Davos en 1999, la détermination de son gouvernement de poursuivre les efforts déjà effectués en vue du développement et de l'intégration des PECO à l'Europe. G. Schröder, « Discours à Davos du $1^{\text {er }}$ février 1999 », Documents d'actualité internationale, 7, $1^{\text {er }}$ avril 1999.

22. Après son élection en 1998, le chancelier Gerhard Schröder (SPD) affirma dans un discours d'investiture devant le Bundestag, et lors du Forum de Davos en 1999, la détermination de son gouvernement de poursuivre les efforts déjà effectués en vue du développement et de l'intégration des PECO à l'Europe. G. Schröder, « Discours à Davos du $1^{\text {er }}$ février 1999 », Documents d'actualité internationale, 7, $1^{\mathrm{er}}$ avril 1999.

23. «Freiheit in Verantwortung. Grundsatzprogramm der Christlich Demokratischen Union Deutschlands » [La liberté en toute responsabilité. Programme de la CDU], programme retenu lors du cinquième congrès du Parti, Hambourg, 20-23 février 1994, p. 86.

24. R. Herzog, "Globalization of German Foreign Policy is Inevitable », German Comments, 39, juillet 1995, pp. 4-12.

25. F. Deloche-Gaudez, «La France et l'élargissement à l'Est de l'Union européenne », Les Études du CÉRI, 46, octobre 1998, pp. 9-10.

26. Le Monde, 2 janvier 1990.

27. A.-M. Le Gloannec, "Mitterrand et l'Allemagne ", French Politics and Society, 9 (3-4), étéautomne 1991, pp.121-129 ; A.-M. Le Gloannec et P. Hassner, «L'Allemagne et la France : deux cultures politiques? », Esprit, 5-221, mai 1996, pp. 44-52.

28. D'après certains interlocuteurs, le risque-pays des marchés est-européens était évalué avant 1994 comme semblable à celui de certains pays africains.

29. MICECO : Mission interministérielle pour la coopération avec l'Europe centrale et orientale ; Cocop : Comité de coopération. Pour une comparaison entre les politiques française et allemande d'assistance à l'Est: E. Tulmets, «L'impact de l'élargissement de l'Union européenne sur la coopération française et allemande à l'Est: quelle gouvernance? ", Revue d'Études Comparatives Est-Ouest, 34 (3), 2003, pp. 111-156.

30. Pour plus de détails sur ce point: R. Hagelberg, «L'Allemagne, la France et le processus d'élargissement de l'Union européenne ", Europe en formation, 3-4, 2002, pp. 65-94.

31. Ce que la citation suivante confirme: « [l'Allemagne a] une vision davantage romantique, ce qui oblige la France à défendre un point de vue plus rationnel [...]. Il ne faut pas perdre de temps pour faire progresser les projets défendus par le couple franco-allemand en matière de construction européenne, avant que l'Allemagne n'ait la tentation de s'affirmer en tant que telle et de jouer un rôle personnel.", "Audition du ministre délégué aux Affaires européennes $\mathrm{M}^{\text {me }}$ Guigou, par la commission des Affaires étrangères de l'Assemblée nationale - 3 octobre 1991 », in Ministère des Affaires étrangères, La Politique étrangère de la France, Textes et documents, septembreoctobre 1991, Paris, MAE, 1991, pp. 93-94.

32. «Discours prononcé par M. le Président de la République à l'invitation de la Berliner Pressekonferenz à l'hôtel Bristol-Kempinski, Berlin, 19 septembre 1991 ", in MAE, 1991, op. cit., p. 56.

33. Commission européenne, Vers une association plus étroite avec les pays d'Europe centrale et orientale. Communication de la Commission au Conseil en vue de la réunion du Conseil européen à Copenhague les 21 et 22 juin 1993, SEC (93) 648 final, Bruxelles, 18 mai 1993, p. 5.

34. Au sujet du débat sur le pouvoir de l'UE, voir: H. Sjursen (éd.), «What Kind of Power? European Foreign Policy in Perspective », Journal of European Public Policy, 13 (2), mars 2006. 
35. PHARE : Pologne-Hongrie, Aide à la reconstruction économique (règlement du Conseil $n^{\circ}$ 3906/89). À l'origine exclusivement destiné à la Pologne et à la Hongrie, le programme a, par la suite, été étendu à l'ensemble des pays d'Europe centrale et orientale candidats à l'adhésion.

36. Commission européenne, Preparation of the Associate Countries of Central and Eastern Europe for Integration into the Internal Market of the Union, COM (95) 163 final, 3 et 10 mai 1995.

37. Sur cette distinction, voir: K. Smith, "The Use of Political Conditionality in the EU's Relations with Third Countries : How Effective? », EUI Working Paper, 97/7, 1997 / European Foreign Affairs Review, 3, 1998, pp. 253-274.

38. Entretiens DG Élargissement, Bruxelles, avril 2003, juin 2004.

39. Commission européenne, «Agenda 2000, For a Stronger and Larger Union », COM (97) 2000 final, 5/97, 1997; Commission européenne, Guidelines for Phare Programme Implementation in Candidate Countries, 1998-1999, 1998 ; Commission européenne, Towards the Enlarged Union. Strategy Document and Report on progress made by each of the Candidate Countries on the Path to Enlargement, $\operatorname{COM}$ (2002) 700 final ; SEC (2002) 1400-1412, 2002.

40. Sur cette "nouvelle méthode», voir: E. Tulmets, "L'adaptation de la méthode ouverte de coordination à la politique d'élargissement de l'UE : l'expérience des jumelages institutionnels en Estonie et en Hongrie ", Politique européenne, 18, hiver 2005.

41. TACIS : Technical Assistance to the Commonwealth of Independent States, Assistance technique pour la Communauté des États indépendants, règlement du Conseil n 1279/96.

42. Bundeskabinett, «Gesamtkonzept zur Beratung beim Aufbau von Demokratie und sozialer Marktwirtschaft in den Staaten Mittel- und Osteuropas sowie der GUS », Kabinettbeschluß, 18 mars 1992 (non publié).

43. L'acquis communautaire désigne l'ensemble des règlements, directives et décisions de l'UE, ainsi que la jurisprudence de la Cour de justice des Communautés européennes.

44. Les jumelages institutionnels (en anglais Twinning) sont venus completer en 1997 la palette d'instruments du programme PHARE pour renforcer les capacités institutionnelles des États candidats. Ils consistent en l'envoi pour une durée de deux ans d'experts des États membres (ministères, agences gouvernementales...) chargés de conseiller leurs homologues des États tiers sur la reprise de l'acquis.

45. ISPA: Instrument for Structural Policies for Pre-Accession, Instrument pour les politiques structurelles de pré-adhésion, règlement 1267/99.

46. SAPARD: Special Assistance Programme for Agriculture and Rural Development, Programme spécial d'assistance pour l'agriculture et le développement rural, règlement 1268/99.

47. L. Yaker, « Est/Ouest-Nord/Sud : quel avenir pour la coopération multilatérale ? ", Cadmos, 13 (50), été 1990, pp. 72-87 ; B. S. Ngoy, «Les nouvelles relations Est-Ouest et leurs incidences sur la coopération CEE-ACP », Africa Development, 18 (3), 1993, pp. 21-36.

48. E. Barbé, «Balancing Europe's Eastern and Southern Dimension », in J. Zielonka (éd), Paradoxes of European Foreign Policy, La Hague/Londres/Boston, Kluwer Law International, 1998, pp. 117-129.

49. En effet, certains paragraphes des rapports produits par la Fondation Bertelsmann pour le compte de la Commission ont été repris mot pour mot dans les textes stratégiques du Partenariat euro-méditerranéen rénové. Entretien avec un membre de la Fondation Bertelsmann chargé de la politique méditerranéenne, 2001.

50. C. Visier, L'État et la coopération, la fin d'un monopole: l'action culturelle française au Maghreb, Paris, l'Harmattan, 2003.

51. Face à l'intérêt croissant de l'Allemagne pour le Sud, certains auteurs n'ont d'ailleurs pas hésité à clamer que l'Allemagne devenait progressivement un "pays méditerranéen ». V. Perthes, «Germany Gradually Becoming a Mediterranean State », Euro-Mediterranean Study Commission, Paper 1, février 1998, www.euromesco.net. 
52. Document cité par K. O. Lang, M. Falkowski, Gemeinsame Aufgabe. Deutschland, Polen und die Ukraine im sich wandelnden Europa / Wspolne zadanie. Polska, Niemcy i Ukraina w przeobrazajacej sie Europie, Warszawa, Institut Spraw Publicznych, 2004.

53. Bertelsmann Foundation and Center for Applied Policy Research (éd.), Thinking Enlarged. The Accession Countries and the Future of the European Union. A Strategy for Reform by the Villa Faber Group on the Future of the EU, octobre 2001.

54. T. Beichelt, « The Impact of Eastern Enlargement on the Common Foreign and Security Policy of the European Union », papier présenté à l'International Studies Association, "Dynamics of World Poli-tics. Capacity, Preferences and Leadership», Honolulu, USA, 1-5 mars 2005. Voir aussi : Commission européenne, Enlargement and the European Neighbourhood Policy, speech by Joe Borg, SPEECH/04/237, 2004 ; Commission européenne, « Beyond Enlargement : Commission shifts Euro-pean Neighbourhood Policy into higher Gear », press release IP/04/632, 12 mai 2005.

55. Commission européenne, "The Second Northern Dimension Action Plan, 2004-06", Commission Working Document, COM (2003) 343 final, Brussels, 10 juin 2003. Voir aussi : H. Haukkala, «The Northern Dimension : A Presence and four Liabilities ?», in R. Dannreuther (éd.), European Union Foreign and Security Policy. Towards a Neighbourhood Strategy, Londres/New York, Routledge, 2004, pp. 98-117 ; D. Lynch (éd.), EU-Russian Security Dimensions, occasional paper $\mathrm{n}^{\circ}$ 46, Institut d'études de sécurité, Paris, juillet 2003, pp. 42-60 (www.iss-eu.org/occasion/ occ46e.html).

56. Le budget du programme Transform s'élevait à $300000 \mathrm{DM}$ par an jusqu'en 1995 $(1$ euro $=1,95 \mathrm{DM})$, date à laquelle il a commencé à diminuer. Ce programme, clos en 1998-2000 pour les pays candidats, a continué à exister exclusivement pour ces trois pays sous une forme moins coordonnée.

57. 1 euro équivaut à environ $1,95 \mathrm{DM}$.

58. P. Guillen, «L'Allemagne unie et l'Europe de l'Est : 1990-1995 », Revue d'histoire moderne et contemporaine, 42 (4), octobre-décembre 1995, pp. 677-686 (p. 682).

59. Le Conseil des États de la mer Baltique a été créé en 1992 à l'initiative des gouvernements allemand et danois pour susciter la coopération entre les pays nordiques (Finlande, Norvège, Islande, Danemark), les pays baltes (Estonie, Lettonie, Lituanie), la Commission européenne, l'Allemagne, la Pologne et la Russie. L'objectif est de renforcer le développement économique entre États membres et non membres (candidats ou tiers) de l'UE et de garantir la sécurité par des moyens autres que militaires.

60. La coopération des pays du Danube est réalisée dans plusieurs domaines, notamment au niveau environnemental ("Coopération régionale des pays du Danube dans le cadre du programme international hydrologique de l'UNESCO»), entre l'Albanie, l'Allemagne, l'Autriche, la Bosnie-Herzégovine, la Bulgarie, la Croatie, la Hongrie, l'Italie, la Macédoine, la Moldavie, la Pologne, la République tchèque, la Roumanie, la Serbie-Monténégro, la Slovaquie, la Slovénie, la Suisse et l'Ukraine.

61. Voir à ce sujet le dossier sur les politiques d'énergie à l'Est paru dans Osteuropa : « Europa unter Spannung. Energiepolitik zwischen Ost und West », Osteuropa, Heft 9-10, 2004.

62. Werner Weidenfeld (éd.), Beyond EU Enlargement. Strategy Paper, Gütersloh, Bertelsmann Foundation Publishers, 2001.

63. R. Prodi, "A Wider Europe. A Proximity Policy as the Key to Stability », SPEECH/02/619, Bruxelles, 2002.

64. Entretien avec un membre de la DG Élargissement, Task Force Neighbourhood, Commission européenne, Bruxelles, juin 2004.

65. Commission européenne, Wider Europe-Neighbourhood: A New Framework for Relations with our Eastern and Southern Neighbours, communication to the Council and the Parliament, 11 mars 2003, COM (2003) 104 final, 2003 ; Commission européenne, Paving the Way for a New Neighbourhood Instrument, Communication COM (2003) 393 final, $1^{\mathrm{er}}$ juillet 2003. 
66. Il s'agit de a) l'espace économique commun (économie et environnement), b) l'espace commun de liberté, de sécurité et de justice, c) l'espace commun de coopération dans les domaines de la sécurité extérieure (gestion de crise, non-prolifération), d) l'espace commun de recherche, d'éducation et de culture.

67. A. Zagorski, « La Russie et l'Allemagne : continuité et changements », Russie. NEI. Visions, 6 (a), septembre 2005. www.ifri.org.

68. R. Del Sarto et T. Schumacher, «From EMP to ENP: What's at Stake with the European Neighbourhood Policy towards the Southern Mediterranean?», European Foreign Affairs Review, 10, 2005, pp. 17-38.

69. MEDA (règlement du Conseil $n^{\circ} 1488 / 96$ ) et MEDA II (règlement du Conseil $n^{\circ}$ 2698/2000) : Mesures d'accompagnement financières et techniques à la réforme des structures économiques et sociales dans le cadre du partenariat euro-méditerranéen. Les bénéficiaires sont le Maroc, l'Algérie, la Tunisie (Maghreb) ; l'Égypte, Israël, la Jordanie, l'Autorité palestinienne, le Liban, la Syrie (Machrek) ; la Turquie, Chypre et Malte ; la Libye a un statut d'observateur lors de quelques réunions.

70. Commission européenne, European Neighbourhood Policy Strategy Paper, Communication from the Commission COM (2004) 373 final, 12 mai 2004 ; Commission européenne, Communication from the Commission to the Council on the Commission Action Plans under the European Neighbourhood Policy, COM (2004) 795 final, 9 décembre 2004 ; EU Press Release, « European Neighbourhood Policy : The Next Steps », IP/05/236, Bruxelles, 2 mars 2005.

71. J. Solana, «Une Europe sûre dans un monde meilleur. Stratégie européenne de sécurité », Bruxelles, 12 décembre 2003.

72. Entretiens DG Élargissement, Task Force Neighbourhood, Commission européenne, Bruxelles, juin 2004.

73. Le président de la Commission Barroso et le chancelier autrichien Schüssel ont confié à Michel Barnier la mission de rendre un rapport sur cette question en juin 2006.

74. Commission européenne, European Neighbourhood Policy Strategy Paper, Communication from the Commission COM (2004) 373 final, 12 mai 2004 (notre traduction).

75. Les priorités couvrent différents domaines: dialogue politique et conduite des réformes; commerce et mesures préparant les partenaires à se rapprocher du marché intérieur de l'UE ; justice et affaires intérieures; énergie, transport, société de l'information, environnement, recherche et innovation; politique sociale et contacts de personnes à personnes (projets culturels, etc.).

76. Pour Simon Nuttall, la cohérence en matière de politique étrangère se définit à trois niveaux: 1) la cohérence horizontale entre les trois piliers de l'UE; 2) la cohérence institutionnelle entre les processus décisionnels communautaire et intergouvernemental ; 3) la cohérence verticale entre la politique de l'UE et celle des États membres, en particulier en matière de mise en œuvre. S. Nuttall, « Coherence and Consistency », in C. Hill et M. Smith (éds.), International Relations and the European Union, Oxford, Oxford University Press, 2005, pp. 91-112.

77. Commission européenne, European Neighbourhood Policy Strategy Paper, op. cit., 2004.

78. P. M. Kaczynski et P. Kazmierkiewicz, « European Neighbourhood Policy : Differentiation and Political Benchmarks », EuroMeSCo, Paper $n^{\circ}$ 44, septembre 2005. www.euromesco.net.

79. Bertelsmann Foundation (éd.), Thinking Enlarged..., op. cit., 2001.

80. W.-D. Eberwein et M. Ecker-Ehrhardt, Deutschland und Polen : eine Werte und Interessen-gemeinschaft ? Die Elitenperspektive, Opladen, Leske + Budrich, 2001.

81. K.-O. Lang et M. Falkowski, Gemeinsame Aufgabe...., op. cit., 2004.

82. Malgré le changement de gouvernement en 2005 , cette conception est toujours présente dans les discours récents de politique étrangère : «L'Ukraine, avec des voisins de chaque côté, sera capable de servir de pont entre l'UE et la Russie ", Steinmeier Frank-Walter, "Germany, the 
European Union and Ukraine - Partners in Europe ", discours du ministre fédéral des Affaires étrangères à l'Académie Mohyla à Kiev à l'occasion de sa visite en Ukraine le 28 février 2006, p. 7. 83. Un accord signé en 2005 entre G. Schröder et V. Poutine a pour but de construire un gazoduc en mer Baltique pour transporter dès 2010 du gaz russe directement vers l'Allemagne en évitant les pays de transit. Voir : "Posten im Aufsichtsrat. Schröder : "Integer" oder "instinktlos" ?", Frankfurter Allgemeine Zeitung, 12 décembre 2005 ; A. Schwabe et C. Volkery, « Neuer Job. Schröder verrubelt seinen Ruf », Spiegel online, 12 décembre 2005 (www.spiegel.de).

84. A. Zagorski, « La Russie et l'Allemagne : continuité et changements », op. cit., 2005.

85. La politique du «3+3» envisage une coopération entre les trois États baltes (Estonie, Lettonie, Lituanie) et les trois pays du Caucase du Sud (Arménie, Azerbaïdjan, Géorgie). Voir par exemple: Bureau du Président de Géorgie, «Joint Press conference of the Georgian and Lithuanian Presidents ", Briefings, 10 octobre 2005. www.president.gov.ge.

86. Entretien avec un membre de la Task Force Voisinage, DG Élargissement, Bruxelles, juin 2004. 87. Ce discours nécessite d'ailleurs des justifications lors de chaque visite officielle de personnalités allemandes dans des pays arabes. V. Perthes, "Germany and the Middle-East ", Working Paper FG6, Stiftung Wissenschaft und Politik, juin 2005. www.swp-berlin.org.

88. Les territoires palestiniens concentrent une large part de la politique bilatérale allemande de coopération dans cette région (532 millions d'euros actuellement), en plus de l'apport financier allemand aux programmes de développement des Nations unies et de la Banque mondiale et des $23 \%$ de participation allemande au budget de développement de l'UE. Source : ministère des Affaires étrangères allemand, http://www.auswaertiges-amt.de/www/en/aussenpolitik/ regionalkonzepte /nahost/friedensprozess_html (janvier 2006).

89. "Germany offers Middle East Peace Talk», The Gardian, 21 août 2001, http:// www.guardian.co.uk/israel/Story/0,2763,540295,00.html.

90. Voir à ce sujet : M. Overhaus, H. Maull et S. Harnisch (éds.), « German Foreign Policy and the Middle-East Conflict ", German Foreign Policy in Dialogue, 3 (7), mai 2002. www.deutscheaussenpolitik.de; H. Fiedler, Deutschland, Europa und der Nahe Osten. Zeitdiagnosen, Band 5, LIT Verlag, 2003.

91. Ibid, p. 6. La feuille de route a été retenue dans la résolution $1515 \mathrm{du}$ Conseil de sécurité des Nations unies du 19 novembre 2003.

92. V. Perthes, « Germany and the Middle-East », op. cit., 2005, p. 2.

93. Même si l'Allemagne est souvent un des trois premiers fournisseurs des pays méditerranéens, cette région représente seulement $3 \%$ du commerce extérieur allemand. Ibid.

94. En 2006, 2514 soldats allemands étaient présents au Kosovo (KFOR), 969 en BosnieHerzégovine (EUFOR), 206 en Méditerranée (OAE) et 11 en Géorgie (UNOMIG). Source : Bundeswehr, mars 2006, cité par: T. Wiegold, «Im Dschungel-Kampf », Focus, 11, 13 mars 2006, p. 32 .

95. Entretiens DG Relex, Politique de voisinage, Commission européenne, avril 2006.

96. TAIEX: Technical Assistance Information Exchange Unit (Unité d'échange d'informations d'assistance technique).

97. Sur les jumelages, voir par exemple : F. Bafoil (dir.), « Les instruments de l'élargissement de l'Union européenne », Critique internationale, 25, octobre 2004 ; E. Tulmets, « The Introduction of the Open Method of Coordination in the European Enlargement Policy : Analysing the Impact of the PHARE/Twinning Instrument", European Political Economy Review, 3 (1), hiver 2004. www.eper.org.

98. Voir M. Notorsky, "Polish and Spanish Visions of the European Neighborhood: Competing or Complementary Interests for the EU Foreign Policy?", papier présenté à la conférence "European Union and its New Neighbourhood: Different countries, Common Interests ", Mykolo Romerio Universitetas, Vilnius, 20-21 octobre 2005. 
99. La France a récemment remporté des projets de jumelages en Serbie avec la Pologne, la Slovénie et la Slovaquie. Entretien à la DG Élargissement, Commission européenne, Bruxelles, mars 2006.

100. Sur le rôle des fondations politiques dans la politique étrangère allemande, voir par exemple les travaux de Dorota Dakowska.

101. Par exemple : Discours d'O. Rehn sur l'élargissement aux pays des Balkans, Agence Europe, «EU/Enlargement: The Commission pleads for "Consolidation" of Enlargement Process...", Bulletin Quotidien Europe, 9065, 10 novembre 2005, p. 2 ; E. Landaburu, « From Neighbourhood to Integration Policy: Are there Concrete Alternatives to Enlargement?», conférence CEPS « Revitalising Europe », Bruxelles, 23 janvier 2006.

102. Z. Laïdi, La norme sans la force : L'énigme de la puissance européenne, Paris, Presses de Sciences Po, 2005.

\section{RÉSUMÉS}

La chute du mur de Berlin a suscité bien des interrogations sur la place de l'Allemagne et de sa politique étrangère en Europe. Pour certains analystes, l'Allemagne risquait d'affirmer de nouveau son rôle sur la scène internationale, alors que depuis 1945 elle avait été contenue par les structures multilatérales euroatlantiques et avait développé une politique étrangère fondée sur ses ressources civiles. En réalité, la politique extérieure allemande continue à se définir dans un contexte multilatéral et européen, qu'il s'agisse de la politique d'élargissement à l'Est ou de la toute récente politique de voisinage de l'Union européenne. Loin de représenter uniquement une contrainte, le multilatéralisme européen prend désormais la forme d'un multi-bilatéralisme principalement fondé sur des ressources civiles, ce qui ouvre la possibilité pour l'Allemagne, comme pour d'autres états membres, d'élargir les domaines de leur politique extérieure.

The fall of the Berlin wall has given rise to new questions, on the place of the reunified Germany in Europe and of its foreign policy. Some analysts felt there was a risk that Germany would reaffirm its role on the international stage whereas from the end of World War II, it had been contained by its integration into multilateral Euro-Atlantic structures and had developed a foreign policy based on civilian power. In fact Germany's positions in the enlargement policy and in the recent neighbourhood policy of the European Union show that its foreign policy continues to be defined in a multilateral and European context, similar to that of other EU member states. Far from representing a constraint, European multilateralism, conceived originally as a method of guaranteeing peace and stability, now takes the form of a multi-bilateralism, mainly based on civilian means, which opens ways for Germany, as well as for other member states, to enlarge the scope of their foreign policy.

\section{INDEX}

Mots-clés : élargissement, politique et voisinage, politique extérieure allemande

Keywords : enlargement, European union, german foreign policy, neighbourhood policy

Index géographique : Union européeenne 


\section{AUTEUR}

\section{ELSA TULMETS}

etulmets@yahoo.de

Dans le cadre de son doctorat en science politique, en co-tutelle à l'Institut d'études politiques de Paris et l'Université libre (FU) de Berlin, Elsa Tulmets a travaillé sur la question de la conditionnalité et des nouveaux modes de gouvernance dans la politique d'élargissement de l'Union européenne à l'Est. Elle a été rattachée au Centre Marc Bloch à Berlin pendant quatre ans, et est actuellement Jean Monnet Fellow à l'Institut universitaire européen de Florence. Ses recherches portent sur la manière dont l'expérience de l'élargissement à l'Est a été transférée vers la Politique européenne de voisinage lancée en 2003 par l'Union européenne. Elle a publié récemment des articles dans la Revue d'études comparatives Est-Ouest, dans European Law Journal et dans Politique européenne. 